\begin{tabular}{|l|l|}
\hline$($ (stöhnt)) & $\begin{array}{l}\text { Kommentar bzw. Anmerkungen zu parasprachlichen, } \\
\text { nichtverbalen oder gesprächsexternen Ereignissen; } \\
\text { die Länge der Klammer entspricht im Falle der } \\
\text { Kommentierung parasprachlicher Äußerungen (z.B. } \\
\text { Stöhnen) etwa der Dauer der Äußerung }\end{array}$ \\
\hline @nein@ & lachend gesprochen \\
\hline @(.)@ & kurzes Auflachen \\
\hline @(3)@ & 3 Sek. Lachen \\
\hline$[\ldots]$ & Markierung von Auslassungen von Interviewtext \\
\hline $\begin{array}{l}\text { für biografische In- } \\
\text { terviews zusätzlich }\end{array}$ & $\begin{array}{l}\text { //mhm// Hörersignal des Interviewers, wenn das } \\
\text { „mhm“ nicht überlappend ist }\end{array}$ \\
\hline $\begin{array}{l}\text { Groß- und Klein- } \\
\text { schreibung }\end{array}$ & $\begin{array}{l}\text { Hauptwörter werden groß geschrieben, und bei Neu- } \\
\text { ansetzen eines Sprechers/einer Sprecherin am Beginn } \\
\text { eines L („Häkchens“) wird das erste Wort mit Groß- } \\
\text { buchstaben begonnen. Nach Satzzeichen wird klein } \\
\text { weitergeschrieben, um deutlich zu machen, dass } \\
\text { Satzzeichen die Intonation anzeigen und nicht gram- } \\
\text { matikalisch gesetzt werden. }\end{array}$ \\
\hline Zeilennummerierung & $\begin{array}{l}\text { Zum Auffinden und Zitieren von Transkriptstellen ist } \\
\text { es notwendig, eine durchlaufende Zeilennummerie- } \\
\text { rung zu verwenden. }\end{array}$ \\
\hline
\end{tabular}

\title{
4.1 DAS VERHÄLTNIS ZUR SOZIOLOGIE
}

\subsubsection{Viele Wege führen zur Soziologie (Der Weg zur Soziologie)}

Die Eingangsfrage der Interviews wurde als zentral angesehen. Auf diese Frage sollten die Interviewten antworten, „wie sie eigentlich zur Soziologie gekommen" seien bzw. was ihre Motivation dafür war, Soziologie zu studieren. In den Antworten ergab sich eine Bandbreite an Orientierungsmustern, die die Wahl des Studienfachs und dementsprechend auch der Soziologie beeinflussten. Interes- 
santerweise gab es kaum Interviewpartner, die die Soziologie von Anfang ihres Studiums an als ihr primäres Fach im Visier hatten - und nun aber erfolgreich, teilweise auf Lehrstühlen, vertreten sind. Diese Aussagen ließen sich über diverse Soziologiegenerationen hinweg gleichermaßen finden. Das Hineinschlittern in die Soziologie selbst mag zunächst nichts Bahnbrechendes beinhalten. Denn möglicherweise stellt dies nichts Ungewöhnliches für Fächer der Geistes- und Sozialwissenschaften dar. Dennoch steht auf den zweiten Blick eine interessante Aussage hinter der Beobachtung: Zunächst bedeutet das Hineinschlittern in die Soziologie eine Unkenntnis darüber, was das Fach genau repräsentiert. Es könnte mitunter auch an der Fragmentierung, der Diversität sowie der Themenbreite des Faches liegen, dass im Vorhinein wenigen klar ist, um was es in der Soziologie eigentlich geht.

\section{Analyse ausgewählter Interviewsequenzen}

\section{Interview T1, Zeile 4-29}

4 I Sie sind ja auch Soziologe. Wieso haben Sie Soziologie als Ihr primäres $5 \quad$ Fach gewählt?

6 T1 Ahm, ich hab interessanterweise Politikwissenschaft angefangen zu studieren, in [Name der Stadt] damals. das war eine Diplomstudiengang, und ahm im Hauptfach Politikwissenschaft, und Soziologie hatte ich im ersten Nebenfach, und Neuere und Neueste Geschichte im zweiten Nebenfach ahm gewählt und ahm ich hab eigentlich (2) also ich hatte keine klare Vorstellung davon, was Soziologie is, ja ehrlich gesagt, als ich das angefangen habe, irgendwas mit Gesellschaftswissenschaft. aber ich hatte eine klare Vorstellung davon, was Politikwissenschaft is, und dass das relativ nahe an der Praxis von politischer im weitesten Sinne Gestaltung gesellschaftlicher Verhältnisse is und das hat mich eigentlich interessiert. und dann hab ich im Studium gemerkt, dass ahm sozusagen die gesellschaftlichen Verhältnisse zu verstehen, und von da ausgehend das Potenzial zur Veränderung oder Gestaltung überhaupt erst ermessen zu können, dass man dafür eigentlich die Soziologie braucht und die Politikwissenschaft fand ich extrem technisch, institutionalistisch, ahm auch sehr pragmatisch in ner Weise, ja, das hat mir-also da hab ich gar nich das gefunden, was ich gesucht habe. und dann hab ich - im Studium selber hab ich angefangen, obwohl ich jetzt meine Fächerkombination beibehalten habe, sehr viel mehr Soziologie zu studieren, bin in die Soziologie dann gerutscht sozusagen, war da auch $\underline{\mathrm{HiWi}}$ und $\underline{\mathrm{Tu}}$ $\underline{\text { tor }}$, habe da zwei Profs kennengelernt, die ich faszinierend fand ahm einem 
26

27

28

29

bin ich dann später hier in [Name des Ortes] wieder begegnet, als ich hier berufen war und genau von da bin ich dann so in die Soziologie reingekommen während des Studiums und genau, dann hab ich eben meine Dissertation dann nachher in [Name des Ortes] auch im soziologischen Feld gemacht.

\section{Formulierende Feininterpretation}

Thema: Studienwahl (Z. 4-10)

Im ersten Abschnitt geht der Interviewte auf die Frage ein, die ihm die Interviewerin gestellt hat. Er verweist auf die irrtümliche Annahme, dass Soziologie sein „primäres“ Fach war. Er stellt klar, dass er ,,interessanterweise“ Politikwissenschaft im Hauptfach gewählt hatte und Soziologie nur als Nebenfach.

Thema: Vorstellung von Soziologie (Z. 10-15)

Im Weiteren geht er darauf ein, dass er zunächst „keine klare Vorstellung“ von Soziologie hatte, damals sei Soziologie für ihn ,irgendwas mit Gesellschaftswissenschaft". Daraufhin geht er auf sein Hauptfach, die Politikwissenschaft, ein. Hier betont er, dass er von ihr eine „klare“ Vorstellung hatte. Er führt aus, was ihn besonders an der Politikwissenschaft gereizt hat, nämlich dass diese aus seiner Sicht nah an der „Praxis von politischer im weitesten Sinne Gestaltung gesellschaftlicher Verhältnisse“ war. Genau das, meint er, habe ihn ,interessiert“.

\section{Thema: Vorstellung Soziologie und Grenzziehung zu Politikwissenschaft} (Z. 15-21)

Erst mit der Zeit hat er dann gemerkt, dass er die Soziologie „,braucht““. Denn nur diese scheint geeignet, zunächst die ,gesellschaftlichen Verhältnisse zu verstehen und das „Potenzial für Veränderung“ abzuschätzen. Im Folgenden spricht er über die Politikwissenschaft, die ihm dann sehr „technisch“ und ,institutionalistisch“"vorkam und die nicht das war, was er ,gesucht“ hatte.

\section{Thema: Weiterer Karriereverlauf (Z. 21-29)}

T1 beschreibt, dass er daraufhin zwar nicht sein Studium gewechselt, aber „sehr viel mehr" Soziologie studiert hat. Er legt zudem dar, dass er bereits als Studierender in der Soziologie angestellt war und zwei Professoren für ihn wichtige Figuren waren. So sei er dann in die Soziologie ,gerutscht“. Zudem weist er auf seinen jetzigen Karrierestatus hin - Professor - und stellt heraus, dass er außerdem auch im ,soziologischen Feld“ promoviert hat. 


\section{Reflektierende Interpretation}

Thema: Studienwahl (Z. 4-10): Erzählung

T1 reagiert auf die Frage, wie er eigentlich zur Soziologie gekommen sei, mit einer Gegendarstellung. Er bezieht sich zu Anfang auf die Politikwissenschaft, zeigt damit also auch eine deutliche Distanzierung zwischen Soziologie und Politik. Mit dem Wort ,interessanterweise“ markiert der Interviewte gleich zu Anfang, dass es nicht selbstverständlich ist, sondern eine Besonderheit (,,interessant), dass er zunächst Politikwissenschaft studiert hat.

\section{Thema: Vorstellung von Soziologie (Z. 10-15):}

\section{Beschreibung mit der Hintergrundkonstruktion im Modus einer Bewertung}

T1 geht in seiner Äußerung zunächst in die Defensive: Der Zusatz „,ehrlich gesagt“ deutet fast auf eine Rechtfertigung hin, mit der er seine Unkenntnis darüber schildert, was Soziologie ist. Er verstärkt dies rhetorisch mit dem lapidaren Ausdruck ,,irgendwas mit Gesellschaftswissenschaft“ und distanziert sich damit auch von der Soziologie, wie er sie zum damaligen Zeitpunkt erfahren hat. Mit der Äußerung, dass er eine „klare Vorstellung“ (im Interview stimmlich betont) von Politikwissenschaft hatte, macht er eine Dramaturgie auf, mit der er eindrucksvoll den Unterschied zwischen Soziologie und Politikwissenschaft markiert. Er drückt in diesem Zusammenhang auch eine Wertung aus, denn gerade hier schildert er, dass ihn eben genau diese Vorstellung von Politikwissenschaft, die er hatte, auch interessiert habe. Mit der Beschreibung, was ihn interessiert hat, nimmt er auch Stellung zu seinen Motiven für ein Studium: Es ging ihm um die Gestaltung politischer und, wenn auch rhetorisch etwas abgeschwächt, gesellschaftlicher Verhältnisse.

\section{Thema: Vorstellung Soziologie und Grenzziehung zu Politikwissenschaft}

\section{(Z. 15-21): Bewertung mit der Hintergrundkonstruktion im Modus}

\section{der Erzählung}

T1 schwenkt nun in seiner Dramaturgie um: Denn die von ihm zunächst aus Unkenntnis stiefmütterlich behandelte Soziologie bekommt im weiteren Verlauf eine prominente Rolle: Er „braucht“ die Soziologie, um „die gesellschaftlichen Verhältnisse zu verstehen, um von da ausgehend das Potenzial zur Veränderung oder Gestaltung überhaupt erst ermessen zu können“. Hier rudert er wieder einen Schritt zurück. Denn es geht ihm nun nicht mehr primär um Gestaltung, sondern um das Verstehen und um Potenziale der Veränderung. Diese Aussage im Zusammenhang mit Soziologie ist interessant, da sie in diesem Kontext ein Alleinstellungsmerkmal der Soziologie darstellt, ohne das eine solche Leistung nicht möglich wäre. Er fertigt damit eine Selbstbeschreibung der Soziologie an, indem 
er sich auf die Exklusivität des Gegenstands bezieht und eine Sicht auf den Gegenstand formuliert, die man mit keiner anderen Disziplin hat. Soziologie wird als eine Wissenschaft konstruiert, mit der die Gesellschaft im Ganzen verstanden und das Potenzial der Veränderung abgeschätzt werden kann. Hier zieht er auch eine klare Grenze zur Politikwissenschaft. In der Bewertung fallen Begriffe wie „technisch“, ,institutionalistisch“ und „pragmatisch“, die alle in diesem Kontext negativ konnotiert sind und im Widerspruch zur Sichtweise der Soziologie stehen. Er beschreibt sich selbst als jemanden, der in der Politikwissenschaft auf der Suche nach etwas war, was er dort nicht gefunden hat. Damit deutet er an, dass er schon vor seinem Studium eine gewisse Absicht zur Veränderung der Gesellschaft hatte, wie er zudem im ersten Absatz bereits verdeutlicht hat. Aus den Ausführungen ist ebenfalls zu erkennen, dass er lediglich die Politikwissenschaft und nicht sein zweites Nebenfach „Neuere und Neueste Geschichte“ als Objekt der Grenzziehung gewählt hat. Das lässt vermuten, dass er die Politikwissenschaft als der Soziologie vergleichbarer einstuft als das Fach Geschichte.

Thema: Weiterer Karriereverlauf (Z. 21-29):

\section{Erzählung mit der Hintergrundkonstruktion im Modus einer Bewertung}

Es ist letztlich konsequent, wenn T1 nun die Geschichte weiter ausführt. Nachdem er seinen Standpunkt klar gemacht hat, weist er darauf hin, dass er dann sehr viel mehr Soziologie studierte, ohne das Studium seiner beiden anderen Fächer abzubrechen. In diesem Abschnitt hebt er zudem die Rolle der professionellen Sozialisation für seinen weiteren Karriereverlauf in der Soziologie hervor. Zunächst in seiner Rolle als HiWi und Tutor und dann über die Bekanntschaft zweier „faszinierender" Professoren. Interessant ist in diesem Abschnitt eine weitere Aussage: Er konstatiert, dass er durch ein intensives Studium in die Soziologie rein „gerutscht“ ist. Nach diesen vielen absichtsvollen Schritten im Vorfeld wirkt der Ausdruck ,hineinrutschen“ defensiv und zufällig.

Interview T11, Zeile 4-72

4 I Zuerst hätte ich mal ein paar biografische Fragen, und zwar würde mich $5 \quad$ interessieren, wie Sie eigentlich zur Soziologie gekommen sind?

6 T11 Wie die Jungfrau zum Kinde, aber ich hab zunächst mal nicht Soziologie studiert, so- sondern ich hab zunächst Jura studiert, äh ich ähm (2) zu Jura bin ich gekommen, also (2) oh Gott, lange Geschichte irgendwie, also ich bin- ich bin ein Kind sozusagen ähm ähm des sogenannten roten Jahrzehnts der 70er-Jahre, ähm das heißt ähm ich bin äh in den 70er-Jahren sozialisiert worden, ältere Brüder, die sozusagen so die- die Spätzünder der 68er 
waren, äh bin dann- bin sehr frühzeitig mit 13, 14 Jahren äh politisch sehr aktiv gewesen. [...] ich bin also in einer extrem politisierten, auch konfrontativen äh äh Atmosphäre als Jugendlicher aufgewachsen, hab mich politisch engagiert. es gab dramatische Ereignisse. äh zwei Mitschüler aus meiner Klasse und einer aus der Parallelklasse äh sind in den Terrorismus auch geraten, äh haben auch jemand umgelegt, äh gehörten der Bewegung 2. Juni an, äh und die waren dann auf einmal aus unserer Klasse verschwunden, äh und dann waren die im Gefängnis. dann kam die Isolationshaft und das haben wir alles mitbekommen und das führte dazu, dass viele von uns ähm ähm ähm also Jura studiert haben, äh wir wollten Rechtsanwälte werden und äh so. ähm also ich hatte das Gefühl, dass ich äh über die Gesellschaft alles weiß, was ich wissen muss, äh äh und hab da-war daher nicht auf den Gedanken gekommen, Sozialwissenschaften zu studieren, sondern dachte, ähm dass die Revolution eher Rechtsanwälte braucht und hab das dann eben auch studiert, äh und hab dann aber auch-war dann auch in einer sehr bewegten und ähm linken bis linksradikalen Szene in meiner Studentenzeit und hab dann auch Anwälte kennengelernt bis hin zu Otto Schily damals, ähm die-die das Profil so hatten, das mir so vorschwebte als junger Mann und hab dann gesehen, dass das keine Existenz ist, die ich führen wollte, äh weil aus meinem-aus meiner-aus meiner-aus meiner Sicht heraus sozusagen es notwendig gewesen wäre, eine Art von bürgerlicher Doppelexistenz aufzubauen mit einem bürgerlichen- mit einermit einer bürgerlichen Existenz, ähm ähm (2), die äh-die-die-die gewissermaßen den Lebensunterhalt sichert, integriert in die bürgerliche Geschäftswelt etc., wo man Verträge abschließt etc. und einer anderen Seite, wo man dann politisch riskante Dinge tut usw. das wollte ich nicht, hatte das Gefühl, (holt Luft) hatte aber auch das Gefühl, ich muss jetzt irgendwie, wenn ich- wenn- hab das dann abgebrochen, hatte das Gefühl aber, ich brauche auch meinen Eltern gegenüber, ehrlich gesagt ich brauch's nicht, hätte vielleicht- ich hätte vielleicht äh eine- eine Auszeit nehmen können irgendwie so, hab aber das Gefühl gehabt, ähm ich müsste gleich mit ner Alternative kommen. und die nächstlegende Alterna- nächstliegende Alternative war die, auf das zurückzukehren, von dem ich meinte voller Arroganz, dass ich es sowieso schon kann, nämlich Gesellschaftslehre, Soziologie usw. [...] dann merkte ich aber natürlich, das waren so bestimmte Eindrücke, damals in meiner Studienzeit war das vor allen Dingen [Name der Person], der war damals noch Marxist //I: hm// später ist er dann Grüner geworden, später ist er dann Neoliberaler geworden, äh als er dann nach [Name des Ortes] ging. damals war er marxistisch orientiert, auch selber 
51

52

53

54

55

56

57

58

59

60

61

62

63

64

65

66

67

68

69

70

71

72

erklärtermaßen marxistisch orientiert, ähm und da hab ich dann gelernt, äh dass ich sozusagen ein dummer arroganter@(.)@ überheblicher junger Mann war, [...] äh dass da noch ein ziemlicher Abstand war und es noch ne Menge zu lernen gab. so- so bin ich in die Soziologie gekommen, ähm und äh gewissermaßen aus ner Verlegenheit heraus, ja, äh aus einer-aus einer starken politischen Motivation heraus, äh weil ich über- über mehr als ein Jahrzehnt, 15 Jahre lang, äh sozusagen und vielleicht sogar noch länger, also stark einem bestimmten linken politischen Milieu auch verhaftet war und äh auch im Laufe meines Soziologiestudiums ähm war ich eigentlichwollt ich eigentlich nicht äh Soziologe werden, sondern äh woll-also eigentlich wollte ich dann Journalist werden und nur der Zufall hat dazu geführt, ähm dass ich nicht äh sozusagen eine Karriere bei Spiegel gemacht habe etc., [...]. das heißt um ein Haar, ja, um ein Haar wär' ich in den Journalismus, wenn das- es war dann ein bisschen frustrierend, dass es irgendwie nicht geklappt hat, äh und auch dann war es wiederum, also sonst wär' ich einfach im Journalismus gelandet, [...]. und äh und dann war es bisschen der Zufall, dass nach dem- nach dem Diplom und nach dem Fehlschlag, [...] ähm äh dass ich dann äh so ne-so einen Bezug also zu so einer Uni-Stelle kriegte, ja, äh und dann hatte ich auf einmal- und- und dannund zuerst als Hilfskraft usw. usf. dann war auf einmal der Kontakt da, äh der-der Kontakt war auch schon vorher da, aber dann gab's so auf einmal Opportunities, ja, und dann bin ich reingekommen.

\section{Formulierende Feininterpretation}

Thema: Studienwahl (Z. 4-29)

Gleich im ersten Satz reagiert der Befragte auf die Frage mit dem Ausdruck „Wie die Jungfrau zum Kinde“. Er fährt fort, dass er zunächst Jura studiert habe. Er wählte dieses Fach, da er eine sehr politisierte Jugend hatte und selbst ,politisch sehr aktiv gewesen“ ist. Insbesondere nimmt er Bezug auf Ereignisse in seiner Schule und auf politische Aktivitäten seiner Klassenkameraden. Da er sich selbst, was Gesellschaft angeht, als sehr erfahren eingestuft hat, war für ihn ein Studium der Sozialwissenschaften nicht interessant. Hier nimmt er expliziten Bezug auf die vermeintliche Notwendigkeit der Revolution und geht auf einige Vorbilder wie Otto Schily ein. 


\section{Thema: Selbstbeschreibung Lebensführung (Z. 29-39)}

Hier beschreibt der Interviewte, dass ein Anwaltsleben nicht seiner Lebensvorstellung entsprochen hätte, da dieses zu sehr in der „bürgerlichen Geschäftswelt““ verankert sei, was letztlich zum Abbruch des Studiums führte.

\section{Thema: Vorstellung Soziologie (Z. 39-54)}

Als „nächstliegende Alternative“ wird dann die Gesellschaftslehre in Betracht gezogen. Der Interviewte ging von der Vorstellung aus, dass es sich hier um Fächer handelt, die er sowieso schon beherrschte. Während des Studiums änderte sich diese Einstellung, auch durch den Kontakt mit bestimmten Vorbildern in der Disziplin. Hier beschreibt sich der Interviewte im Rückblick mit einem Lachen als ,dummen arroganten überheblichen jungen Mann“.

\section{Thema: Weiterer Karriereverlauf (Z. 54-72)}

Er schließt seine Ausführungen damit, dass seine Wahl der Soziologie ,aus ner Verlegenheit“ heraus erfolgte. Er begründet diese Wahl mit einer starken ,politischen Motivation“, schließt jedoch sofort an, dass er ursprünglich Journalist werden wollte und es nur dem „Zufall“ und einem „Fehlschlag“ zu verdanken sei, dass er dann eine Stelle an der Universität bekam. Weitere „Opportunities“ folgten, durch die er dann in die Soziologie „reingekommen“ ist.

\section{Reflektierende Interpretation}

Thema: Studienwahl (Z. 4-29):

\section{Erzählung mit der Hintergrundkonstruktion im Modus der Begründung}

T11 leitet seine Erzählung mit einer Redensart ein. Diese Redensart steht dafür, zu etwas unverhofft oder ohne eigenes Zutun zu kommen. Er distanziert sich damit von einer absichtsvollen Auswahl seines Studienfachs Soziologie. Zunächst begründet er in einer biografischen Erzählung, die mit einer Zuordnung zu einer Generation und ab dem Jugendalter beginnt, seine Studienfachauswahl Jura. Er greift in seiner Begründung auch auf seine frühe Sozialisation zurück, die nicht erst mit dem Studium einsetzte, sondern mit seiner Kindheit als eines von mehreren Geschwistern und seiner politisierten Schulzeit begann. Er beschreibt, wie insbesondere diese intensiven persönlichen Erfahrungen ihn dazu veranlasst haben, Jura zu studieren, und dass diese Studienwahl in enger Verbindung zu seiner Weltanschauung stand. Er betont, dass er Jura nicht primär aus Interesse studiert hat, sondern, weil er Jura „brauchte“, um ein bestimmtes Ziel zu erreichen: die Revolution. Seine Studienfachwahl wird also als Mittel zum Zweck begründet. Entscheidend ist der Bezug zu einem radikal linken Milieu, in 
dem er sich während seiner Studierendenzeit bewegte, und zu bekannten Persönlichkeiten, die ihn beeindruckten.

Thema: Selbstbeschreibung Lebensführung (Z. 29-39):

Erzählung mit der Hintergrundkonstruktion im Modus der Begründung

Hier fertigt er nun eine Selbstbeschreibung an, wie er sich sein Leben als Ideal vorgestellt hat, und distanziert sich von eben diesem Bild. Er beschreibt sich selbst als radikalen Menschen, der, seinen Idealen verpflichtet, nicht mit Kompromissen leben möchte. Das hätte aus seiner Sicht ein Leben bedeutet, das er als „,bürgerliche Doppelexistenz“ beschreibt: auf der einen Seite ein bürgerliches Leben führen und auf der anderen Seite „politisch riskante Dinge“ tun. Diese Selbstbeschreibung als moralisch integren und politisch kompromisslosen Menschen setzt er dann konsequent fort, indem er schildert, dass er das Studium abgebrochen hat.

Thema: Vorstellung Soziologie (Z. 39-54):

Begründung mit der Hintergrundkonstruktion im Modus der Erzählung

Diesen Abschnitt beginnt T11 mit einer sehr starken Form der Begründung. Im Zentrum steht hier die Erläuterung, weshalb er auf die Soziologie gekommen ist. Denn der Abbruch des Studiums scheint ihn unter Zugzwang gesetzt zu haben. Während dieser Begründung bricht er immer wieder ab, fängt neu an und bildet keinen vollständigen Satz. Das deutet auf eine innere Zerrissenheit hin, die er möglicherweise immer noch mit dieser Entscheidung verbindet. Das Studium der Soziologie scheint er demnach nicht aus Interesse, sondern aufgrund einer günstigen Gelegenheit ergriffen zu haben - entsprechend wird die Soziologie als „nächstliegende Alternative“ beschrieben, also in gewissem Sinn wie Jura als Mittel zum Zweck. Der Zweck war jedoch ein anderer: Der war nicht die Revolution, sondern die Notwendigkeit, möglichst schnell eine Alternative zu finden, die wenig Aufwand erfordert. Von dieser Einstellung distanziert er sich in seiner Erzählung mehrfach, indem er auf sich als ,arroganten jungen Mann“ rekurriert. Auch hebt er die Rolle von Vorbildern hervor.

\section{Thema: Weiterer Karriereverlauf (Z. 54-72):}

Begründung mit der Hintergrundkonstruktion im Modus der Erzählung Auch in diesem Abschnitt fällt auf, dass der Interviewte im Modus der Begründung verbleibt, wenn er von seinem weiteren Karriereverlauf spricht. Er argumentiert hier, dass er während seines Studiums nicht vorhatte, Soziologe zu werden. Das betont er mehrmals in seinen gesamten Ausführungen. Es ist eine doppelte Ablehnung der Soziologie: zunächst zugunsten des Jurastudiums und dann 
zugunsten seines Berufswunsches, in den Journalismus zu gehen. In beiden Fällen war die Soziologie eher der „Notnagel“, Soziologie aus „Verlegenheit“. In seiner Selbstbeschreibung spielt zudem seine „starke politische Motivation“ eine herausragende Rolle. Auch diese erwähnt er mehrmals. Dennoch erscheint es, als wären die von ihm erdachten Alternativen wie Jura und Journalismus jeweils für ihn passender als die Soziologie. Er bezeichnet seine Ablehnung an der Journalistenschule als „Fehlschlag“. Wie T1 umschreibt auch T11 seinen Zugang zur Soziologie als „dann bin ich reingekommen“ und zwar über unterschiedliche „Opportunities“. In seiner Selbstbeschreibung wählt er die Soziologie aufgrund von Möglichkeiten und aus Verlegenheit.

Interview T2, Zeile 18-51

T2 [...] also insofern ist das ne Frage, die man sich immer wieder stellen muss ähm, ich hab mir natürlich auch schon ahm gestellt, warum Wissenschaftler, eigentlich muss man sagen, ja ahm ahm, vielleicht erstmal warum Soziologe, oder wie Soziologe oder so was äh::mm (2). also ich (2) es ist ne gute Frage, warum eigentlich das, also ich kann nur sagen, dass es schon relativ früh war bei mir und das mag mit familiären Verstricktheiten äh zusammenhängen, das war sozusagen eine problematische Familiensituation und äh äh äh, da hab ich mich immer so ein bisschen rausgenommen und ähh bin sozusagen in die Beobachterposition und bin dann abgedriftet und äh äh äh äh da hat sich irgendwie ein grundsätzliches Interesse an äh äh, an so Fragen wie wie wie ist denn dieses soziale Zusammenleben@(2)@ähorganisiert, irgendwie herausgebildet. ähm, (2) am Anfang war das noch nicht mal die Soziologie, sondern es war es eher die Anthropologie, also also die Ethnologie, ich dachte die ist noch viel allgemeiner als die Soziologie, dass es halt sich immer auf die restlichen Kulturen äh äh äh bezieht und äh dann hab ich auch Ethnologie angefangen und parallel äh war noch die Soziologie aber als Nebenfach die Soziologie nur und äh dann musst ich aber erfahren, dass äh äh dass (2) in der Ethnologie, zumindest wo ich war, das war damals in [Name der Stadt], äh äh die an Verallgemeinerungen überhaupt kein Interesse hatten, ja. //I: hm// da gings um den einzelnen Stamm und wat weiß ich, das war für mich äh äh sowas von uninteressant und gleichzeitig, zur gleichen Zeit hab ich dann Theorieseminare in der Soziologie besucht und da wars genau anders rum in gewisser Weise nur Generalisierungen und so. und irgendwann war dieses Spannungsverhältnis nicht mehr auszuhalten, sodass ich diese Ethnologie gekippt hatte und dann bin ich in die Soziologie gegangen und hab dann stiefmütterlich eigentlich die 
44

45

46

47

48

49

50

51

Politikwissenschaft dazu genommen, dachte auch immer, das ist eine Bindestrichsoziologie ähhh, die Politikwissenschaft,demwarnichtso@(3)@ ähm, da ging es stärker um ah normative Fragen auch noch noch ahm und oweniger um so umo- ich bin dann sehr früh in die empirische Forschung reingekommen über unterschiedliche Forschungsprojekte, DFG-Projekte ähmm also als Hilfskraft, ob das nun Transkribierarbeiten waren oder die Teilnahme an Interpretationssitzungen, das ist eigentlich, was ich heute auch noch so ein bisschen mache.

\section{Formulierende Feininterpretation}

Thema: Studienwahl (Z. 18-34)

Der Interviewte hebt hervor, wie wichtig es sei, sich zunächst zu fragen, warum er sich für die Soziologie interessiert. Er begründet das mit seinem familiären Hintergrund, in der er aufgrund einer ,problematischen Familiensituation“ immer wieder in die „Beobachterposition“ „,abgedriftet“ ist. Hier hat sich sein Interesse dafür entwickelt ,wie denn dieses soziale Zusammenleben organisiert ist“. Er stellt klar, dass er anfangs jedoch überwiegend in der Ethnologie verankert war.

Thema: Grenzziehung Soziologie - Ethnologie - Politikwissenschaft (Z. 34-47) Im Anschluss stellt er heraus, dass seine Vorstellung von Ethnologie nicht dem entsprach, was er in seinem Studium vorfand. Er war viel stärker an Verallgemeinerungen interessiert, als dies in der Ethnologie verhandelt wurde. In der Soziologie hat er die Erfahrung gemacht, dass es dort nur um „Generalisierungen“ geht. Aufgrund eines empfundenen Spannungsverhältnisses zwischen den beiden Fächern wechselt er in die Soziologie und nimmt „stiefmütterlich“ die Politikwissenschaft hinzu, in der es jedoch stärker um normative Fragen geht.

\section{Thema: Weiterer Studienverlauf (Z. 47-51)}

Er stellt dar, dass die qualitative Sozialforschung, die er als studentische Hilfskraft kennenlernte, auch heute noch sein Schwerpunkt ist.

\section{Reflektierende Interpretation}

Thema: Studienwahl (Z. 18-34):

\section{Erzählung mit der Hintergrundkonstruktion einer Beschreibung}

T2 begründet sein Interesse, ähnlich wie T11, aus seiner persönlichen Biografie heraus. Dennoch setzt er nicht, wie T11, eine Selbstbeschreibung eines politisierten Jugendlichen ein. Vielmehr rekurriert er auf die „Beobachterposition“, die er aufgrund familiär schwieriger Situationen eingenommen hat. Er beschreibt, wie 
er durch ein solches „Abdriften“ eine Außenposition gegenüber seiner Familie eingenommen hat. Diese Außenposition abstrahiert er dann, indem er über die Außenposition in der Familie auf das Interesse an Außenpositionen in der Gesellschaft kommt, also daran, wie ,gesellschaftliches Zusammenleben organisiert ist". Dies ist eine Selbstbeschreibung, die er ausgehend von seinem Interesse an grundsätzlichen Fragen darstellt. Doch auch er findet, wie die anderen Interviewteilnehmer, nicht sofort seinen Weg zur Soziologie. Seine Studienfachwahl fällt zunächst auf die Ethnologie, in der er seine Interessen vertreten glaubt.

Thema: Grenzziehung Soziologie - Ethnologie - Politikwissenschaft (Z. 34-47):

Erzählung mit der Hintergrundkonstruktion einer Beschreibung

T2 zieht nun eine deutliche Grenze zwischen Soziologie und Ethnologie. Soziologie wird hier stark mit „Verallgemeinerungen“ in Zusammenhang gebracht, Ethnologie mit dem „,einzelnen Stamm“. Er fertigt eine Selbstbeschreibung an, die die Soziologie mit der Qualität der Verallgemeinerung auszeichnet. Er betreibt auch Grenzarbeit zur Politikwissenschaft. In einem ersten Schritt entgrenzt er sie zunächst, indem er sie als „Bindestrichsoziologie“ bezeichnet. Der zweite Aspekt in der Grenzarbeit sind - im Gegensatz zur Soziologie - die ,normativen Fragen“. Es fällt auf, dass er hier den Satz abbricht und seinen Gedanken nicht weiter ausführt.

Thema: Weiterer Studienverlauf (Z. 47-51): Erzählung

In seiner biografischen Erzählung führt er die Geschichte fort. Er verfolgt seinen Weg zur Soziologie, auf dem er sich für die empirische Sozialforschung entscheidet, die er bis heute betreibt.

Interview T8, Zeile 4-31

$\begin{array}{ccc}4 & \text { I } & \text { [...] Ähm wie sind Sie eigentlich zur Soziologie gekommen? } \\ 5 & \text { T8 } & \text { Ich ähm wollte eigentlich ursprünglich ähm Lehramt Sozialkunde und Reli- } \\ 6 & & \text { gion machen //I: hm// ich hab'angefangen, Theologie zu studieren in [Name } \\ 7 & & \text { der Stadt] und da mir klar war, weil ich bin Katholik, das äh Zölibat wär } \\ 8 & & \text { nix so richtig für mich gewesen, und ähm da dacht'ich, na ja, wie kann ich } \\ 9 & & \text { Theologie studieren und ne Berufsperspektive haben, na ja gut, dann werd' } \\ 10 & & \text { ich Lehrer //I: hm// und da war die Idee, also ich muss gestehen, dass die } \\ 11 & & \text { Theologie im Zentrum stand //I: hm// und die Soziologie kam dazu als Mög- } \\ 12 & \text { lichkeit, dann Sozialkundelehrer zu werden. und als ich dann anfing, Sozio- } \\ 13 & \text { logie zu studieren, das ging relativ schnell, war mir klar, also ich hab' } \\ 14 & \text { Theologie und Soziologie studiert und hatte irgendwie relativ viel Philoso- }\end{array}$


phie gemacht, dann kam irgendwie so ne Hegel-Geschichte da rein, dass ich sehr viel Hegel las und dachte na ja, Hegel ist ja eigentlich Soziologie //I: hm// und ähm dann hatte ich ein bisschen Probleme, was äh äh so die dogmatische Struktur der Theologie betraf und dann hab' ich die Soziologie aufgegeben und hab' dann, ähh die Theologie aufgegeben und nur noch auf die Soziologie (geguckt). [...] ähm na ja das ist schon klar durch die Theologie, mich hat eigentlich ähm die verständnis- und orientierungswissenschaftliche Seite der Soziologie interessiert //I: hm// also so ein bisschen so ne Durkheim-Frage, gibt es irgendeine Art von Wissenschaft, die aus unserer gesellschaftlichen Existenz heraus Ideen unseres Lebens ableiten kann und das hat mich eigentlich immer interessiert. Also die Soziologie als so ne Art von indirekter Ethik, als ne Art und Weise, auch aus empirischen Grundlagen her Lebensweisen und Möglichkeiten von Lebensweisen zu verstehen. also mich hat weniger interessiert ähm am Anfang zu sagen, ich will irgendwie spezifische Phänomene //I: hm// ähm entschlüsseln, sondern mich hat schon immer diese Orientierungsfunktion an der Soziologie interessiert, die natürlich auch nah an der Theologie ist.

\section{Formulierende Feininterpretation}

Thema: Studienwahl (Z. 5-20)

T8 beschreibt, dass er zunächst Sozialkunde und Religion auf Lehramt studieren wollte. Das Lehramt ließ das Studium der Theologie mit einer Berufsperspektive zu, ohne später im Zölibat leben zu müssen. Die Soziologie sah er zunächst als Ergänzung zur Theologie. Dann beschreibt er, wie er über die Philosophie zur Soziologie gekommen ist und dass er auch zunehmend „Probleme“ mit der „dogmatischen Struktur“ der Theologie hatte und deshalb nur noch auf die Soziologie fokussiert hat.

\section{Thema: Vorstellung Soziologie (Z. 20-31)}

T8 beschreibt, dass ihn - ursprünglich getrieben von der Theologie - besonders die orientierungswissenschaftliche Seite der Soziologie interessiert hat. Er beschreibt die Soziologie als eine Art von indirekter Ethik, aus „empirischen Grundlagen her Lebensweisen und Möglichkeiten von Lebensweisen zu verstehen“. Das interessiert ihn an der Soziologie stärker als ,irgendwie spezifische Phänomene“. Er stellt die Theologie mit der Soziologie gleich, indem er sagt, dass „diese Orientierungsfunktion“ „,auch nah an der Theologie ist“. 


\section{Reflektierende Interpretation}

Thema: Studienwahl (Z. 5-20):

Erzählung mit der Hintergrundkonstruktion im Modus der Begründung

Wie die vorherigen Interviewpartner schildert auch T8, dass sein ursprünglicher Fokus nicht auf der Soziologie lag. Wie bei T11 werden auch hier zwei Hindernisse geschildert, die die Soziologie als nächstgelegene Alternative scheinen lassen, um die eigenen Vorstellungen an das Studienfach zu erfüllen. Zunächst musste T8 aufgrund des Zölibats auf eine Hilfskonstruktion ausweichen - Lehramt Sozialkunde und Theologie -, um die Aussicht zu haben, von der Theologie leben zu können. Die Soziologie wurde dann ergänzend zur Theologie gewählt, um den Berufswunsch umzusetzen. Dann jedoch kam ein zweites Hindernis, das wiederum eine Entscheidung hervorrufen musste. T8 kam mit der „dogmatischen Struktur" der Theologie nicht zurecht, weshalb er sich dann vollends auf die Soziologie konzentrierte. Im Gegensatz zu T1 oder T2 führt er dann jedoch keine Grenzziehung, sondern eine Entgrenzung der Soziologie durch: Zunächst vereinnahmt er die Philosophie durch die Soziologie durch den Ausspruch „Hegel ist ja eigentlich Soziologie“. Durchaus hätte das auch anders herum geschehen können: Soziologie ist ja eigentlich Hegel, also Philosophie. Er entscheidet sich für die erste Variante und inkorporiert damit auch sein Interesse an der Philosophie in die Soziologie.

Thema: Vorstellung Soziologie (Z. 20-31):

Begründung mit der Hintergrundkonstruktion im Modus der Beschreibung Diese Entgrenzung setzt er nun im weiteren Verlauf fort: Denn auch hier begründet er sein Interesse an der Soziologie durch die Theologie. Dabei rekurriert er auf die verständnis- und orientierungswissenschaftliche Seite der Soziologie. Seine Selbstbeschreibung verweist bereits auf seine Motivation, Soziologie zu betreiben. Diese begründet er zunächst mit einem soziologischen „Schwergewicht“: Émile Durkheim und der „Durkheim-Frage“. Damit bettet er sein Interesse direkt in einen Kontext der großen Soziologen ein. Stark grenzt er sich von einem anderen Interesse in der Soziologie ab, nämlich spezifische Phänomene zu „entschlüsseln“. Er bezieht sich auf die Theologie, indem er die Soziologie als eine Art ,indirekte Ethik“ beschreibt und deren Orientierungsfunktion betont, also die normative Frage, wie man aus ,unserer gesellschaftlichen Existenz heraus Ideen unseres Lebens ableiten kann". 


\section{Orientierungsmuster „Weg zur Soziologie“ oder Soziologie in the making}

\section{Der Weg zur Soziologie als Weg der Umwege}

Es zeigte sich in der Analyse der Focussierungsmetaphern, dass keiner der Interviewten vor dem Studium die Absicht besaß, den Schwerpunkt auf die Soziologie zu legen. Auch verdeutlichte die Analyse, dass kein Interviewpartner eine konkrete Vorstellung davon besaß, was Soziologie sein könnte. ${ }^{2}$ Soziologie war vielmehr oft zweite oder dritte Wahl und wurde verknüpft mit Vorstellungen einer bestimmten Lebensführung oder politischen Einstellung bzw. stellte eine Alternative zum ursprünglichen Berufswunsch dar. Alle vier Interviewten beschreiben, wie sie letztlich aufgrund von „Opportunities“ in die Soziologie „hineingerutscht" sind, und zwar über eine Beschäftigung als studentische Hilfskraft oder später im Anschluss durch die Anstellung als wissenschaftlicher Mitarbeiter.

\section{Soziologie als variables Konzept der Selbstbeschreibung}

Die Soziologie zeichnet sich also durch eine Interpretationsoffenheit aus: Die Begründungen für die Wahl des Studiums fielen jeweils unterschiedlich aus. Von Abgrenzung bis hin zu Enttäuschung in Hinblick auf die erstgewählten Studienfächer - die Wahl der Soziologie kam aus ganz unterschiedlichen Gründen zustande. Der Orientierungsrahmen, der sich durchgängig in den Interviewpassagen gezeigt hat, kann als Interpretationsoffenheit der Soziologie bezeichnet werden.

Diese Interpretationsoffenheit speiste sich eben nicht aus konkreten Vorstellungen die Soziologie betreffend, sondern aus persönlichen Interessen, die bereits vor dem Studium bestanden. Die Soziologie ist so gesehen ein flexibles Konzept, das in unterschiedlicher Weise mit diversen Selbstbeschreibungen gefüllt wurde. Diese beinhalteten Grenzziehungen wie auch Entgrenzungen zu anderen Fächern. Begründet werden diese mit unterschiedlichen semantischen Konstruktionen, die kognitiv-epistemische wie sozial-politische Argumentationsmuster umfassen.

Eine Selbstbeschreibung, die sich an kognitiv-epistemischen Charakteristika orientiert, führt Argumente an, die sich auf die Soziologie als Disziplin und auf ihre Wissenseigenschaften beziehen. Die Anforderungen an die Soziologie wa-

2 In weiteren, hier nicht analysierten Interviews wurde der Weg in die Soziologie als „aus der Not geboren“ (T9) bzw. als eine Mischung aus „Verlegenheit und Gelegenheit" (T7) dargestellt. 
ren insbesondere, dass Aussagen abstraktionsfähig und „generalisierbar“ sein sollten. T2 konstruiert in diesem Zusammenhang eine Außenposition, die er als Beobachtungsmoment für die Soziologie geltend macht. T2 betreibt Grenzarbeit zur Ethnologie und zur Politikwissenschaft, die ihm als zu detailreich und zu „,normativ“ erscheinen. Der Weg zur Soziologie ist im Fall von T2 als Orientierungsmuster zu sehen, das sich als „Konstruktion von Außenpositionen und deren Generalisierbarkeit" beschreiben lässt. Auch T8 bewegt sich mit seinem Orientierungsmuster „Soziologie als indirekte Ethik“ auf der kognitiv-epistemischen Ebene, indem er hier letztlich eine Entgrenzung zur Theologie betreibt und die „Ethik“ der Theologie zu einer ,,indirekten Ethik der Soziologie“ umfunktioniert. Normative Aspekte, die ja auch durchaus bei der Theologie zu finden sind, spielen hier jedoch bereits hinein, und zwar im Rückgriff auf die „orientierungswissenschaftliche Seite der Soziologie“. T8 ist wie T2 weniger an der Spezifik einzelner Phänomene interessiert. Auch ihm geht es um Generalisierungen einer Soziologie als Verständnis- und Orientierungswissenschaft.

Die Selbstbeschreibungen von T1 und T11 orientieren sich hingegen stärker an sozial-politischen Kriterien für die Wahl der Soziologie. Ausschlaggebend sind weniger disziplinäre Charakteristika als eine Sinnkonstruktion einer „Soziologie als Potenzial gesellschaftlicher Veränderung“. Sozial-politische Aspekte spielen in der Selbstbeschreibung eine zentrale Rolle, da die Soziologie überwiegend in Zusammenhang mit gesellschaftlich-politischer Veränderung gesehen wird. Dennoch wird diese Rolle in der oben genannten Sinnkonstruktion zunächst zurückgenommen. Denn nicht die gesellschaftliche Veränderung, sondern das Potenzial gesellschaftlicher Veränderung ist für T1 relevant. Potenziale aber führen erst in einem zweiten oder dritten Schritt, vielleicht auch niemals zu realer gesellschaftlicher Veränderung. Im Gegensatz dazu ist bei T11 die Wahl des Fachs Soziologie - obwohl als letzte aller Möglichkeiten - in der Rhetorik stark von einer „politischen Motivation“ geprägt. Soziologie wird in diesem Zusammenhang also als dritte Möglichkeit gesehen, sich politisch auszudrücken - in einer langen Rangfolge von Jura bis hin zum Journalismus.

Um den Orientierungsrahmen der Interpretationsoffenheit der Soziologie zu systematisieren, kann also zusammengefasst werden, dass die Selbstbeschreibungen sowohl von kognitiv-epistemischen Elementen geprägt waren als auch von sozial-politischen. Das, was die Interviewten mit der Soziologie als Disziplin verbanden, war geprägt von biografischen Erlebnissen, persönlichen Erwartungen und verwehrten Chancen. So ist eine biografische Sinnkonstruktion in Hinblick auf den Weg zur Soziologie als „Sociology in the making“ zu interpretieren. Die Interpretationsoffenheit der Soziologie, die aus theoretischer Sicht auf die mangelnde kognitive Konsolidierung des Fachs zurückzuführen ist, bietet al- 
so einen breiten Fundus unterschiedlicher Sinnkonstruktionen. Dieser ermöglicht den Weg zur Soziologie, anders als bei anderen Fächern oder Berufen, die nicht in der Form offenstanden. Die analytische Unterscheidung zwischen epistemisch-kognitiven Aspekten der Selbstbeschreibung und sozial-politischen deutet darauf hin, dass sich die Doppelstruktur der Soziologie bereits in der Auswahl des Fachs zeigt: Eine stärkere Orientierung auf ein „Innen“ der Soziologie, also die Disziplin selbst, zeigt sich in der Angabe von epistemisch-kognitiven Sinnkonstruktionen. Eine Orientierung auf ein „Außen“ in Hinblick auf die Gesellschaft und das Veränderungspotenzial zeigt sich in sozial-politischen Kriterien der Sinnkonstruktionen.

Wie sich an diesem Analyseabschnitt gezeigt hat, gab es aus biografischer Sicht also weder eine übergreifende Identitäts- oder Sinnkonstruktionen „der“" Soziologie noch gab es Bekundungen, dass man schon früh ausschließlich die Soziologie als Betätigungsfeld im Blick gehabt habe. Vielmehr war der Weg zur Soziologie geprägt von Umwegen und Abgrenzungen zu dem, was die Interviewten nicht wollten. Die Soziologie bot aufgrund ihrer Interpretationsoffenheit einen geeigneten Rahmen, in den eigene Sinnkonstruktionen eingefügt werden konnten.

\subsubsection{Die Motivation für Soziologie}

Die Frage „Motivation für Soziologie“ wurde meist ergänzend zu den biografischen Fragen gestellt. Wie wir im ersten Auswertungsabschnitt gesehen haben, sind bereits aus der Studienfachwahl implizite Motivlagen für das Studium der Soziologie abzuleiten. Dennoch wird hier noch einmal bewusst zwischen dem Weg zur Soziologie und der Motivation für Soziologie unterschieden, nicht zuletzt, um abzugleichen, ob es möglicherweise Unterschiede zwischen der Rekonstruktion der biografisch orientierten Orientierungsmuster gibt und der Motivlage, die die Interviewten für die Soziologie angeben, mit der sie aktuell arbeiten. Die Motivlage für die Soziologie wurde je nach Verlauf der Interviews direkt oder indirekt erfragt. So wurden die Interviewpartner(innen) beispielsweise mit einer offenen Frage dazu aufgefordert, zu beschreiben, welche Art von Forschung sie betreiben und warum. Dies ließ Rückschlüsse auf Sinnkonstruktionen über die eigene Arbeit in der Soziologie - ihre Motivation für Soziologie - zu. 


\title{
Analyse ausgewählter Interviewabschnitte
}

\author{
Interview T6, Zeile 99-150
}

99 I Ähm Sie haben grade vorhin auch gesagt, die Art der Soziologie, die Sie be-

100 treiben,

$101 \quad \mathrm{~T} 6 \quad \mathrm{~L} j a$

102 I wie würden Sie denn die Art der Soziologie beschreiben, die Sie betreiben?

103 T6 Also es kommt drauf an. Wollen Sie's von außen nach innen oder von innen $104 \quad$ nach außen beschrieben haben, aber also von außen nach innen gesehen $105 \quad$ ähm, man würde zunächst mal sagen ist im Bereich der sogenannten quali106 tativen Sozialforschung, ähm aber ich würd' da so nicht ansetzen. //I: hm// 107 also ich würd' anders anfangen und sagen, wa-was ist ganz eindeutig, ich 108 äh seh mich in der sogenannten verstehenden Soziologie, also würd' ich Weber, Schütz ganz brav, ja, äh und dann kommen eben Verunreinigungen rein, aber das ist wieder was anderes, aber so das, die Logik ist die verstehende Soziologie, die-der theoretische Ansatz, an dem ich auf Teufel komm raus festhalte, ist Handlungstheorie. also ich lass da gar nicht mit mir verhandeln, äh ich sag auch immer ich versteh' zum Beispiel Systemtheorie einfach nicht, ja, ich versteh sie nicht, ähm die meisten Menschen denken, dies sei eine boshafte Bemerkung von mir und glauben nicht, dass ich's wirklich nicht versteh', ich versteh' aber nicht, was die macht. ähm so und dann würd' ich die Forschung und bei mir ist sehr sehr wichtig, also ich mach'bei mir geht's immer um die Vermit-Ver- um irgendeine Verbindung von theoretischer Überlegung und empirischer Arbeit, ja und äh von der Forschung her würd' ich mich nie der qualitativen Sozialforschung zuwenden, sondern was- was wir machen ist interpretative Sozialforschung [...] also interpretative Sozialforschung ähm datenerhebungstechnisch ist das einfach Ethnografie, was- was ich ganz wesentlich mache, also nicht nur, aber äh meistens ist es- sind es ethnografische Designs, [...], ähm also es heißt, einfach würd' ich grundsätzlich ein- äh ein- eine Mixtur aus Beobachtung, aus mit Leuten reden, aus- die Dinge aus dem Feld sich angucken //I: hm// ja das ist [Name der Person] ähm, die ist-die kommt aus der Quanti-Ecke und ähm die sagt immer, das sei ne ziemlich wilde Art, wie-wie-wie wir so diese Methoden miteinander, weil mir ist das wurscht, ja, mir geht's einfach drum, ich will irgendwas wissen und dann nehm' ich irgendwas, was ich methodisch brauchen kann, aber ich-ich-mich interessiert die Methode nicht an sich, ja. aber man muss die- die Methoden natürlich können, das ist klar. ähm das ist die Datenerhebungsseite und äh auf der andern Seite äh die Da- 

ten hermeneutisches Vorgehen, äh mit ganz ganz wenig Aufweichungen [...] das heißt keine phänomenologische Philosophie, ja, nicht schwer verständliche Dinge, sondern äh ne methodische Herangehensweise, äh Dinge zu betrachten, ja und äh ich sag immer das hilft gegen Betroffenheitslyrik. wenn man so arbeitet wie wir, ja und ganz nah an den Feldern ist //I: hm// äh und sich da drauf einlässt und de-dezidiert affirmativ, wir sind dezidiert unkritisch, ja wir sind immer auf der Seite von unsern Forschungssubjekten, aber wir helfen ihnen nicht, also wir sind nicht emanzipativ, gar nicht, ja //I: hmhm//wir gucken kalt lächelnd zu wie jemand ertrinkt, wenn sie so wollen, wie in diesem Zen-Beispiel, ja, wo der-Sie kennen das, geht ein Zen-Mönch einen Fluss entlang, im Fluss äh treibt ein Mann und ruft verzweifelt ich ertrink, ich ertrinke und der Mönch sagt, es tut mir leid, ich kann dir nicht helfen, ja, ja, wir können dir nicht helfen, nichts //I: hm// äh aber wir sind sehr affirmativ //I: hm// ähm und damit man dann nicht anfängt, Betroffenheitslyrik zu produzieren, ja und so, da hilft die Phänomenologie als strenge-als strenge Methode der Datenreinigung so würd 'ich das mal sagen, ja.

\section{Formulierende Feininterpretation}

Thema: Situierung in der eigenen Disziplin (Z. 103-116)

Zunächst macht T6 eine Innen-Außenbeschreibung der Soziologie auf. Er beschreibt sein Vorgehen von innen nach außen, indem er sich selber in der verstehenden Soziologie und in der Handlungstheorie verortet und sich von Ansätzen wie der Systemtheorie radikal abgrenzt.

\section{Thema: Beschreibung der eigenen Forschung (Z. 116-133)}

Seine Forschung beschreibt T4 als eine Verbindung zwischen theoretischen Überlegungen und empirischer Arbeit. Sein Fokus liegt hier weniger auf einer stringenten Methode, sondern darauf, dass er ,irgendwas wissen“ will und dann „,irgendwas“ nimmt. Diesen Teil bezeichnet er als die Datenerhebungsseite.

\section{Thema: Grenzziehung zum Untersuchungsgegenstand (Z. 133-150)}

Die andere Seite bezeichnet er als die Datenauswertung. Hier bezieht er sich strikt auf das hermeneutische Vorgehen „mit ganz wenigen Aufweichungen“. Diese hilft ihm, gegen „Betroffenheitslyrik“ anzukommen. Dann erläutert er, dass er sich ganz nah am Feld befindet und welches Verhältnis er zu seinen Forschungssubjekten hat. Er bemüht dann eine Parabel, um sein Verhältnis deutlich zu machen. 


\section{Reflektierende Interpretation}

Thema: Situierung in der eigenen Disziplin (Z. 103-116):

Argumentation mit dem Hintergrundmodus einer Bewertung

Zunächst macht $\mathrm{T} 6$ eine eigene Heuristik auf, in der er eine äußere und eine innere Argumentationsschicht definiert. Die äußere Schicht wird als sein methodischer Zugang, die qualitative Sozialforschung, definiert. Den inneren Kern stellt für ihn die „verstehende Soziologie“ dar. Dieses Bild verstärkt er, indem er soziologische Schwergewichte wie Max Weber oder Alfred Schütz anführt. Zudem scheint dies zunächst ein reiner Kern zu sein, zu dem „Verunreinigungen“ kommen. Hier definiert er seinen Zugang über „,reine“ Soziologen, die auch als Klassiker der Disziplin bezeichnet werden können. Dann legt er eine weitere Situierung fest: Er beschreibt sich als Handlungstheoretiker. Auch diese Aussage unterstreicht er, indem er betont, dass er in diesem Punkt „nicht mit sich verhandeln" lässt. Er grenzt sich von der Systemtheorie ab, wofür er kognitive Gründe aufführt (,,ich versteh aber nicht, was die macht").

Thema: Beschreibung der eigenen Forschung (Z. 116-133):Argumentation mit der Hintergrundkonstruktion einer Beschreibung und einer Bewertung

T6 geht genauer auf die Definition seiner eigenen Forschung ein. Interessant ist in diesem Abschnitt, dass er sich in Hinblick auf seine vorhergehenden Aussagen widerspricht. Während er sich im ersten Abschnitt noch der qualitativen Sozialforschung zugeordnet hat, differenziert er hier zwischen qualitativer und interpretativer Sozialforschung. Unter Letztere fasst er vor allem die Ethnografie und die Methode, mit der er vorgeht. Er zeiht eine Grenze zwischen quantitativer und qualitativer Forschung, wobei er die vermeintliche Sauberkeit der Methoden thematisiert. Im Gegensatz zu seiner Situierung in der Soziologie, die als rein und auch akkurat bezeichnet werden kann, positioniert er sich hier viel stärker auf der Seite seiner eigenen Interessen, der sich dann die Methode unterzuordnen hat. Deutlich wird das insbesondere durch den Satz „weil mir ist das wurscht, ja, mir geht's einfach drum, ich will irgendwas wissen und dann nehm' ich irgendwas, was ich methodisch brauchen kann, aber ich- ich- mich interessiert die Methode nicht an sich". Hier kommt sein Hauptinteresse an der Soziologie zum Vorschein: Es ist nicht primär ein methodisches, sondern es geht um die Umsetzung eines Interesses, einer Wissenslücke, die er in Bezug auf bestimmte Phänomene hat. 
Thema: Grenzziehung zum Untersuchungsgegenstand (Z. 133-150):

Argumentation mit dem Hintergrundmodus einer Beschreibung

Nach einer ausführlichen Schilderung seines epistemologischen Zugangs im ersten Abschnitt und seines methodischen Zugangs im zweiten Abschnitt geht es nun um seine Datenauswertung. Er wendet viel Energie auf, diese im Detail zu beschreiben. Zwei Dinge stechen hervor: Wie im ersten Abschnitt rekurriert er auf die „Reinheit“, jetzt in Bezug auf die Auswertungsmethode. Er trennt zwischen Erhebung und Auswertung, da diese im Gegensatz zur Erhebung nicht beliebig ist, sondern ein hermeneutisches Vorgehen mit ,ganz wenigen Aufweichungen". Die Rahmung, die dieses hermeneutische Vorgehen erfährt, ist die Vermeidung von „Betroffenheitslyrik“. T6 beschreibt dies ausführlich. Zunächst ist für ihn Betroffenheitslyrik etwas, von dem man sich distanzieren sollte. Sie entspricht wissenschaftlichen Standards nicht. Wichtig ist für ihn die erfolgreiche Distanzierung von dem Untersuchungsgegenstand, denn gerade die Form der Forschung, die er betreibt, scheint zunächst mit wenig Distanz verbunden zu sein, da sie „dezidiert unkritisch“ und „immer auf der Seite“ der Forschungssubjekte ist. Diese Distanz erzeugt er rhetorisch über zwei Formen: 1. Über die Negation jeglicher Hilfe für die Forschungssubjekte. Er rekurriert auf eine sehr eindringliche Parabel über einen Mönch, der einen Menschen ertrinken lässt, obwohl dieser nach Hilfe ruft. Er konstruiert dementsprechend eine Außenperspektive, die eine Distanzierung von einer Innenperspektive schafft, die er durch die affirmative Haltung in seiner Forschung einnimmt. 2. Über die „Phänomenologie als strenge Methode“, die die Daten von einer eigenen möglichen Betroffenheit reinhalten soll.

\section{Interview T4, Zeile 3-30}

3 I $\quad[. .$.$] zu den Zielen [...] Ihres Forschungsprogramms, und warum Sie diese$ $4 \quad$ Ziele $[\ldots]$ verfolgen?

5 T4 (2) Das ist eine große Frage@(.)@, also das eine ist natürlich, dass man im 6 innertheore- innerwissenschaftlichen Diskurs das beantworten kann, und $7 \quad$ wenn man sich da die Soziologie anschaut, dann gibt's halt wenige, die wirklich [Name des Bereichs] erforschen. [...] also normalerweise haben sie entweder die [Name des Bereichs-] forscher oder die [Name des Bereichs-]forscher, und die Idee hier ist eigentlich, ahm das ebend hier immer wieder zu verbinden, und das wär ebend (.) und daraus ergeben sich ganz viele wissenschaftliche Forschungslücken ○im Prinzipo. (Seufzt) ähm das wär die eine Definition, die andere Definition ist, wenn man Leibniz ernst nimmt und sagt, wir machen problemorientierte Grundlagenforschung, dann 
sind das natürlich schon auch Themen, die gesellschaftlich relevant sind, und praktisch in diesem großen Bereich von [Name des Bereichs] und dem großen Bereich von [Name des Bereichs] haben wir dann schon eher den Fokus auf Fragen da, wo es hapert. [...] also die Fragen, wo man sagt, das ist so das untere Ende der Sozialhierarchie und die muss man halt lösen. man kann natürlich auch eine andere Logik haben, die muss man auch lösen, die Probleme, aber das ist so ein bisschen unser Zugang dazu. (3) und äh, insofern würd ich schon sagen, dass viele unserer Projekte erstmal nicht unbedingt anwendungsorientiert sind, sondern eher grundlagenorientiert sind, aber bei der Problemauswahl, also bei der Fragestellung (4) wir dann eher Ressourcen darauf verwenden, wo wir meinen, da gibt's ein Problem, was es zu lösen gibt oder nich. [...] die Idee ist eigentlich, man will Aufklärung betreiben und man will gestalten. und äh (2) ehrlicherweise war das immer@meine Motivation@,warumich Soziologie studiert habe, und insofern passte das dann mit dem [Name der Organisation] einfach gut, weil das das ist, was man machen soll, aufklären und gestalten.

\section{Formulierende Feininterpretation}

Thema: Situierung in der Soziologie (Z. 5-23)

T4 differenziert in der Beschreibung des Forschungsprogramms in einen ,innerwissenschaftlichen“ Diskurs und in ,,problemorientierte Grundlagenforschung“. Mit diesen Zugängen sind unterschiedliche Fragen verbunden.

Thema: Beschreibung der eigenen Forschung (Z. 23-27)

T4 beschreibt die eigene Forschung und inwieweit die betreffenden Projekte dem einen oder anderen Zugang entsprechen. Hier bringt er zudem eine dezidierte Definition dessen, was die Ziele der Forschung sind.

Thema: Motivation Soziologie (Z. 27-30)

T4 beschreibt konzise seine Motivation für Soziologie.

\section{Reflektierende Interpretation}

Thema: Situierung in der Soziologie (Z. 5-23):

Argumentation mit der Hintergrundkonstruktion einer Begründung

T4 eröffnet, wie T6, seine Situierung mit einer Heuristik. Diese differenziert wie T6 zwischen einem Innen und einem Außen, die als eine Dialektik eingeführt werden. Rhetorisch schafft er Distanz zu der Beschreibung des innerwissenschaftlichen Diskurses, indem er mit seinen Ausführungen in der dritten Person („man“) bleibt. Zudem bleibt er im Konjunktiv: „das wär die eine Definition.“ 
Der Einsatz des Wortes „natürlich“ zeigt an, dass diese erste Definition diejenige ist, die als selbstverständlich gilt. In der Dramaturgie wird nun eine Spannung aufgebaut, was denn die andere Definition sein könnte. Diese wird im Indikativ formuliert: ,die andere Definition ist“. Dies deutet an, dass das die für ihn relevante Definition ist. Im weiteren Satzverlauf bezieht er sich auf Leibniz: „wenn man Leibniz ernst nimmt". Der Name hat Gewicht; immerhin ist Leibniz einer der wichtigsten Universalwissenschaftler des 17. Jahrhunderts. „Problemorientierte Grundlagenforschung“ wird also mit einem „großen“ Namen begründet und legitimiert. Denn es würde im Umkehrschluss all diejenigen diskreditieren, die „Leibniz“ nicht ernst zu nehmen. Hier zieht der Interviewte eine Grenze zwischen einer ,innerwissenschaftlichen“ Definition und einer anderen, die sich auf problemorientierte Grundlagenforschung bezieht. Problemorientierte Grundlagenforschung speist sich aus einem lebensweltlichen Problembezug und zwar „da wo es hapert“.

Thema: Beschreibung der eigenen Forschung, (Z. 23-27):

Argumentation mit der Hintergrundkonstruktion einer Begründung

Im weiteren Verlauf setzt der Interviewte seine Ausführungen zu seiner eigenen Forschung in Bezug. Auffallend ist die lange Pause inmitten seiner Ausführungen. Er positioniert sich auf der Seite der Grundlagenforschung. Die Projekte, die er macht, sind also „nicht unbedingt anwendungsorientiert“. Hier betreibt er klassische Grenzziehung zwischen anwendungsorientierter Forschung und Grundlagenforschung. Er verbindet diese Forschungstypen, indem er die Fragestellung als etwas bezeichnet, wo „wir meinen, da gibt es etwas zu lösen oder nicht“, sie ist auf normativen Annahmen begründet. Dieses Vorgehen in der Problemauswahl jedoch problematisiert er nicht, weil es letztlich zu der von ihm vorgebrachten Argumentation zu problemorientierter Grundlagenforschung passt, die allem voran mit Leibniz argumentiert und begründet wurde. Er geht damit zwar einerseits auf Distanz zu einer möglichen Anwendung, andererseits lässt er Raum für lebensweltliche, normative Zugänge zum Forschungsgegenstand, die in dieser Form dann auch nicht weiter begründungsbedürftig scheinen.

\section{Thema: Motivation Soziologie (Z. 27-30):}

\section{Erzählung mit dem Hintergrundmodus einer Begründung}

Hier geht er auf die Motivation ein, Soziologie zu betreiben, und zwar als Aufforderung: „Das ist es, was man machen soll: aufklären und gestalten.“ Hier zeichnet er ein Bild einer nach außen gerichteten Soziologie, die aufklärend und gestaltend wirken soll. Er bezieht sich auf seine vorhergehenden Aussagen, in 
denen er auf seinen Zugang zu Forschungsfragen eingeht: Es sind Fragen, „die muss man halt lösen“.

Interview T10, Zeile 761-778

761 I Gehört [...] diese Vermittlung der Forschungsergebnisse an die Praxis im 762 weitesten Sinne zu den Aufgaben eines Wissenschaftlers oder einer Wissen763 schaftlerin?

764 T10 Nach meinem Verständnis schon. Das ist der Grund, wieso ich Wissen765 schaft mache. Ich will nicht nur die Gesellschaft verstehen, ich will auch $766 \quad$ wieder in die Gesellschaft hineinwirken. Ich bin ja selber Teil der Gesellschaft. Für mich war immer schon klar, dass das ein Teil davon ist. Wie stark ich da hineinwirke, und wie viel meiner Energie und Aufmerksamkeit auf diesen Aspekt gerichtet ist, versus Verstehen und ein selbstreferenzielles Wissenschaftssystem mit am Leben zu erhalten, das ist eine Sache, wo man gucken muss, an welcher Stelle der Karriere oder des Lebens was ansteht. Aber grundsätzlich, finde ich, gehört das in jedem Fall dazu. Und ich würde mich freuen, wenn es auch institutionell und infrastrukturell stärker gefördert würde, dass wir das machen. Es müssen ganz bestimmt nicht alle partizipativ forschen, aber zu lernen, wie man sich verständlich ausdrückt, auch Menschen außerhalb der Wissenschaft verständlich zu machen, wieso die Arbeit relevant ist und wieso es auch für die Gesellschaft wichtig ist, dass Menschen Wissenschaft machen, ist glaube ich ganz wichtig.

\section{Formulierende Feininterpretation}

Thema: Motivation für Wissenschaft (Z. 764-772)

In diesem Abschnitt beschreibt T10 seine Motivation für Wissenschaft und Möglichkeiten der Umsetzung dieser Motivation.

Thema: Orientierung Wissenschaft (Z. 772-778)

T10 macht deutlich, dass er es grundlegend gutheißen würde, wenn ein solches Verständnis in der Wissenschaft vorherrschen würde.

\section{Reflektierende Interpretation}

Thema: Motivation für Wissenschaft (Z. 764-772):

Argumentation mit der Hintergrundkonstruktion einer Beschreibung Ähnlich wie T4 begründet auch T10 seine Motivation für Soziologie mit einer sozial-politischen Orientierung. T10 bezieht sich jedoch in seiner Begründung zunächst nicht auf die Soziologie, sondern auf die Wissenschaft im Allgemeinen. 
Die Argumentation, die T10 vorbringt, unterscheidet sich maßgeblich von der von T4. Die Motivation wird nicht mit einer prominenten Person begründet, sondern mit der Stellung, die T10 in Bezug auf seinen Untersuchungsgegenstand einnimmt: Er ist selber Teil der Gesellschaft und will deshalb die Gesellschaft nicht nur verstehen, sondern auch in sie hineinwirken. Des Weiteren macht er die Limitierungen eines solchen Hineinwirkens nicht von epistemologischen Faktoren abhängig, wie das bei T6 der Fall war, sondern von strategischen Faktoren der Karrieregestaltung in der Wissenschaft. Ebenfalls angeführt werden institutionelle Aspekte für die Förderung eines Hineinwirkens in die Gesellschaft, die bislang unzureichend ist. T4 nimmt eine Grenzziehung zwischen „Hineinwirken in die Gesellschaft“ und einem ,selbstreferenziellem Wissenschaftssystem" vor.

Thema: Orientierung Wissenschaft (Z. 772-778):

Argumentation mit der Hintergrundkonstruktion einer Beschreibung

T10 zeichnet ein breites Bild von Wissenschaft. Ihm geht es insbesondere darum, die Relevanz von Wissenschaft für die Bevölkerung erkennbar und verständlich zu machen. Damit verbunden sieht er einen Lernprozess, den die Wissenschaftler durchlaufen müssten.

\section{Orientierungsmuster „Die Motivation für Soziologie“}

Die Interviewabschnitte zeichnen sich überwiegend durch den Texttyp der Argumentation aus. Es wurde in den einzelnen Textpassagen vermehrt auf theoretisch-legitimatorisches Wissen zurückgegriffen. Im Allgemeinen zeigte sich auch hier die Interpretationsoffenheit der Soziologie, nämlich dadurch, dass die Interviewten der Soziologie unterschiedliche Eigenschaften zuordneten. Auch machten sie, wie im Abschnitt zuvor, sowohl kognitiv-epistemische als auch sozial-politische Dimensionen in den Selbstbeschreibungen geltend. Während T10 und T4 ähnliche Selbstbeschreibungen anfertigten, indem sie angeben, eine Soziologie betreiben zu wollen, die ,,aufklärt und gestaltet“, war dieses Thema in der Selbstbeschreibung von T6 nicht relevant. Seine Beschreibung der Soziologie legte den Schwerpunkt überwiegend auf epistemisch-kognitive Argumente. Er distanzierte sich von möglicher „Betroffenheitslyrik“, die mit einem Soziologieverständnis einhergehen kann, das auf „Helfen“ ausgerichtet ist.

\section{Die Reinheit der Beschreibung und die Vermeidung von Betroffenheitslyrik}

In der Selbstbeschreibung von T6 dominiert das Prinzip der „Reinheit“ - er bezieht sich in seiner Semantik mehrmals auf mögliche „Verunreinigungen“. Diese 
Reinheit trifft einerseits auf seine Situierung innerhalb der Soziologie zu und andererseits auf die Methode der Datenauswertung (Phänomenologie als Datenreinigung). Interessanterweise gilt das Gebot der „Reinheit“ nicht unbedingt für die Problemwahl. Er begründet diese weder mit einer wissenschaftlichen Methode noch anhand wissenschaftlicher Kriterien. Vielmehr möchte er das untersuchen, was ihn interessiert. Der lapidare Ton, in dem er die Problemauswahl beschreibt (,weil mir ist das wurscht, ja, mir geht's einfach drum, ich will irgendwas wissen und dann nehm' ich irgendwas, was ich methodisch brauchen kann"), deutet darauf hin, dass er keinerlei Rechtfertigungszwang und damit verbunden Selbstvergewisserungsrhetorik darin sieht. Die dahinterliegende ,professional ideology“ einer „science for science sake“ kann diese Forschung begründen, die an intrinsischen Interessen ausgerichtet ist. Wissenschaft ist also nicht an einer Rhetorik der Nützlichkeit, sondern an der Freiheit der Forschung orientiert und bewegt sich ganz in einem selbstreferenziellen Rahmen der Wissenschaft, also im „Innen“.

\section{Die gestalterische Kraft der Soziologie}

Ganz anders verhält es sich mit T4, der sich in der problemorientierten Grundlagenforschung situiert. Diese Situierung bringt eine Selbstvergewisserungsrhetorik in der Selbstbeschreibung mit sich. Dies zeigt sich einerseits darin, dass er eine Rhetorik der Autorität einsetzt: „,wenn man Leibniz ernst nimmt“. Andererseits wird deutlich, dass er sich immer wieder auf die eigene „Wissenschaftlichkeit" rückbezieht, in Form der Leibniz-Referenz und der Aussage, dass ja überwiegend Grundlagenforschung betrieben werde. Durch diese Form der Selbstbeschreibung, die als Legitimierungsrhetorik zu bezeichnen ist, stellt T4 epistemische Autorität her, um die Art der Forschung, die er betreibt, zu legitimieren: ,aufklären und gestalten“. Denn die Motivation für die Art der Forschung, die T4 betreibt, orientiert sich weniger an einer epistemisch-kognitiven Rhetorik einer selbstreferenziellen Wissenschaft als an einer sozial-politischen. Im Gegensatz zu T6 wählt T4 die Forschungsfragen nach seinen eigenen Kriterien der sozialen Problemwahrnehmung, also danach, ,wo es hapert“.

T10 richtet sich mit seiner Motivation, die er als „Hineinwirken in die Gesellschaft" beschreibt, am stärksten an einer Selbstbeschreibung aus, die sich an sozial-politischen Kriterien orientiert. Auffallend ist, dass diese Selbstbeschreibung von wesentlich weniger Rechtfertigungszwang gekennzeichnet ist als die von T4. Ein Hineinwirken in die Gesellschaft entspricht der Rolle eines Wissenschaftlers und ist damit auch legitim. In dieser Selbstbeschreibung geht es weniger darum, ein Hineinwirken in die Gesellschaft über ein „Innen“ zu legitimie- 
ren. Faktoren, die zu einer Einschränkung dieses „Hineinwirkens“ führen, sind strategischer Natur.

Innen- und Außenorientierung als Grenzziehung zwischen Wissenschaft und Nicht-Wissenschaft

Alle Interviewten konstruierten eine Innen- und eine Außenperspektive. Das „Innen“ war jeweils stellvertretend für einen Kern. Dieser Kern wurde von T6 als eine reine Soziologie dargestellt, die sich über die Klassiker als verstehende Soziologie beschreibt. Auch T10 nimmt diese Beschreibung vor, er definiert das „Innen“ im Sinne einer selbstreferenziellen und verstehenden Soziologie. T4 definiert ein „Innen“, das er als „innerwissenschaftlichen Diskurs“ bezeichnet. Die Grenzziehungen zwischen Innen und Außen wurden jeweils unterschiedlich vollzogen.

\section{1) Prinzip der Exklusion oder das Reinheitsprinzip}

Wie T6 die Grenze zwischen Wissenschaft und Nicht-Wissenschaft zieht, kann mit der Erfüllung eines „Reinheitsprinzips“ umschrieben werden. Er weist auf die Gefahr einer „Betroffenheitslyrik“ hin, die er scharf von seiner Ansicht von echter Wissenschaft abgrenzt. Diese Betroffenheitslyrik kann, wie bereits erwähnt, nur durch strenge Kontrolle in der Datenauswertung vermieden werden. Die eigene Betroffenheit, also die eigene Normativität und Empathie, darf die Wissenschaft nicht verunreinigen. In Anlehnung an Gieryn wird hier von einer Grenzziehung nach dem Prinzip der Exklusion gesprochen. Im Gegensatz zu Gieryn, der mit dem Prinzip der Expulsion operiert, wird hier bewusst von Exklusion gesprochen: Alles, was nicht zur „reinen“ Soziologie gehört, muss bereits im Forschungsprozess ausgeschlossen werden. Denn es geht nicht nur darum, unterschiedliche Lager aus der Soziologie auszuschließen, was Gieryn als Expulsion beschreiben würde. Ziel ist zudem, die Innengrenzen in Hinblick auf die Doppelstruktur zu stärken und jegliche Verunreinigung durch eigene Werturteile zu vermeiden, also zu exkludieren. Diese rhetorische Grenzziehung zwischen der Soziologie und ihrem Untersuchungsgegenstand mag sich auch aus der Methode begründen: Dadurch, dass T6 mit der Ethnografie sehr nahe am Gegenstand (,sehr affirmativ“) ist, scheint für ihn die Grenzziehung zwischen Wissenschaft und dem Untersuchungsgegenstand in Form der Exklusion besonders virulent. 


\section{2) Prinzip der Inklusion: Grundlagen- und Anwendungsorientierung als legitime Forschungsorientierung}

Im Fall von T4 ist eine Trennung zwischen grundlagen- und anwendungsorientierter Forschung als eine Hilfskonstruktion anzusehen, um die eigene Forschung zu legitimieren und als zulässige Forschung in einem wissenschaftlichen Kanon zu kennzeichnen: Sie ist zugleich grundlagen- und problemorientiert. Dies ist eine Art von Grenzarbeit, die im Folgenden als Prinzip der Inklusion bezeichnet werden soll. Im Gegensatz zum Prinzip der Exklusion geht es in diesem Fall darum, unterschiedliche Prinzipien der Wissenschaft als legitim gelten zu lassen.

Auch die Grenzarbeit von T10 kann mit dem Prinzip der Inklusion umschrieben werden. Der Unterschied zu T4 ist jedoch, dass diese Form der Grenzarbeit mit wesentlich weniger Selbstvergewisserungsrhetorik verbunden ist. Inklusion wird nicht an vermeintlich wissenschaftliche Kriterien gebunden, sondern an die Forderung einer stärkeren institutionellen und infrastrukturellen Förderung. In Hinblick auf die Soziologie selbst wird also eine Selbstbeschreibung vorgenommen, die keiner innersoziologischen Selbstvergewisserungsrhetorik bedarf.

Deutlich wird aus diesen Ausführungen, dass die Art und Weise, wie Grenzarbeit betrieben wird und wie diese aussieht, immer stark von der Selbstbeschreibung und Positionierung der Interviewpartner abhängt. Denn auch wenn T6 und T10 beide nach dem Prinzip der Inklusion verfahren, sind die Begründungsmuster und Zugänge doch unterschiedlich. Auch konnte hier herausgearbeitet werden, dass die Frage nach der wissenschaftlichen Problemauswahl bei T6 und T4 nicht mithilfe wissenschaftlicher Kriterien begründet wurde. Dabei war die Legitimierungsrhetorik bei $\mathrm{T} 4$ wesentlich stärker ausgeprägt als bei $\mathrm{T} 6$. Dies lag an der eigenen Positionierung der Personen im Feld der Soziologie. Hat sich also T6 von Anfang an in einem „Innen“ der Disziplin situiert, so hat T4 sich in seiner Selbstdarstellung zunächst außerhalb der Grenze eines innerwissenschaftlichen Diskurses gesehen und deshalb eine andere Art von Grenzarbeit betrieben als $\mathrm{T} 6$.

Es zeigt sich, dass sich alle drei Soziologinnen und Soziologen selbst auch als solche definieren. Über unterschiedliche rhetorische Strategien jedoch versuchen sie, Legitimität für ihren Zugang zu Soziologie zu erzeugen, was in unterschiedlichen Strategien der Grenzziehung deutlich wird.

\subsubsection{Soziologie ist... oder: Die Aufgabe der Soziologie}

Im Folgenden werden Textabschnitte untersucht, in denen die Interviewten nach der Aufgabe der Soziologie gefragt wurden. 


\title{
Analyse ausgewählter Interviewabschnitte
}

\author{
Interview T3, Zeile 582-607
}

582 I Denken Sie, dass dieser Praxisdialog zu den Aufgaben von Wissenschaftle-

$583 \quad$ rinnen und Wissenschaftlern gehört?

584 T3 Ich bin nich so normativ, dass ich das sagen würde. Für mich wü- für mich 585 seh ich das so, ich empfinde das als meine Aufgabe (3) ahm, ich wüsste 586 sonst nich, also, wenn ich jetzt sagmal Physikerin wäre, dann wüsst ich, dass es da wirklich so was wie - wir wollen uns jetzt nicht über Grundlagen-

$588 \quad$ forschung ernsthaft streiten - aber da wüsst ich, dass Grundlagenforschung - das is entweder richtig auf dem Stand des Wissens oder nich, das gibt's in den Sozialwissenschaften nicht, es gibt immer Tausende von Zugängen, es gibt nicht richtig oder falsch. ich kann nicht - ich kann einen - ich kann jetzt latourische Zugänge zu STS irgendwie verbessern, oder ich kann die Luhmann'sche Theorie zur Kommunikation nehmen und irgend-irgendwas zufügen. das ändert dann aber nichts zu dem diversen Stand sozialwissenschaftichen Wissens zu dem Thema (1) Kommunikation zum Beispiel, es ändert nichts an der Diversität, an der Vielstimmigkeit, an äh an der Kakofonie. das wird auch immer so bleiben. und ich glaube, ich persönlich kann sozusagen dem Steuerzahler und der Steuerzahlerin, für mich ganz - aber das ist wirklich nur für mich individuell, dem könnte ich nicht erklären, warum ich jetzt ahm an der Verbesserung sagmal von Luhmanns Theorie arbeiten müsste partout oder irgendwelche anderen Gedankenexperimente machen.(3) ich seh das so, ich weiß, wir brauchen ne Philosophie, die also sicherlich nich in der Regel ne- äh - nen engen Zugang zur Praxis hat, wir brauchen das sicherlich, wir brauchen Kunst, wir brauchen alles Mögliche, die explizit nicht praxisorientiert sind und die explizit (2) sozusagen spielerisch und was weiß ich an die Dinge rangehen, aber(2) ich bin nich sicher, ob wir Soziologie als Grundlagenforschung brauchen, ehrlich gesagt.

\section{Formulierende Feininterpretation}

Thema: Aufgabe der Soziologie (Z. 582-597)

In diesem Abschnitt erläutert T3, dass er den Praxisdialog als seine Aufgabe empfindet. Hier grenzt er die Soziologie von der Physik ab und kontrastiert diese Art des Wissens mit der des sozialwissenschaftlichen Wissens, das er als dispers und fragmentiert beschreibt. 
Thema: Soziologie als Grundlagenforschung (Z. 597-607)

Dann folgt die Erläuterung, dass T3 es mit Blick auf den Steuerzahler nicht als legitim ansieht, dass diese Art von Wissenschaft selbstreferenziell ausgeübt wird.

\section{Reflektierende Interpretation}

Thema: Aufgabe der Soziologie (Z. 582-597): Argumentation

Im ersten Abschnitt betont T3 mehrere Male, dass seine Aussage nicht für die gesamte Disziplin zutrifft, sondern dass er eine solche Normativität lediglich auf der persönlichen Ebene beansprucht. Er geht auf die Naturwissenschaften ein und betreibt Grenzarbeit zu den Sozialwissenschaften: Das in den Naturwissenschaften generierte Wissen (Beispiel Physik) ist anderer Art als das der Sozialwissenschaften. Das Wissen in den Naturwissenschaften ist im Gegensatz zu den Sozialwissenschaften entweder wahr oder unwahr, was auch seiner Definition von Grundlagenforschung entspricht. Rhetorisch also unterscheiden sich Sozialund Naturwissenschaften im Grad des Wahrheitsgehalts des Wissens. Die Argumentation erinnert an Whitleys Ansatz, der die Sozialwissenschaften als „fragmented adhocracies“ (Whitley, 1984a; Whitley, 1984b) bezeichnet.

\section{Thema: Soziologie im Dienst der Praxis (Z. 597-607): Argumentation}

In diesem Abschnitt führt T3 die Argumentation zurück auf die Frage der Praxisbezogenheit der Soziologie. In diesem Punkt geht er in seiner Begründung außengeleitet vor. Es geht um die Legitimation der Forschung vor dem Steuerzahler. Hier zweifelt T3 zudem den Status der Soziologie als Grundlagenwissenschaft an, da ein solcher vor dem Steuerzahler nicht zu rechtfertigen ist. Obwohl er diesen Status anderen Disziplinen und Bereichen zugesteht, nimmt er letztlich eine Entgrenzung der Soziologie vor, indem er konstatiert, nicht sicher zu sein, ob ,wir eine Soziologie als Grundlagenforschung brauchen ehrlich gesagt“.

Interview T5, Zeile 311-341

311 T5 (3) Erfolgreicher Transfer wäre (2), wenn ich etwas herausfinde ahm und312 ok das wär ein sehr erfolgreicher Transfer, sehr erfolgreicher Transfer wäre $313 @$ @(.)@ich finde etwas über die Mechanismen heraus, und das wird tatsäch314 lich von Verantwortlichen entweder in der Organisation, in der Gesell315 schaft, im Staat, übernommen und ahm tatsächlich dann daraus ne Policy 316 abgeleitet und die dann implementiert. //I: hm// das wär sehr erfolgreich. erfolgreich finde ich auch schon allein Aufklärung. ähm, die - das kann teilweise auch schon sehr erfolgreich sein, wenn einfach das was ich gefunden 
319

hab - wenn die Leute das in ihren Gedanken das berücksichtigen, oder oder wissen, warum manche Dinge so sind, wie sie sind, dass das nicht zufällig, gottgegeben oder ähnliches ist, sondern dass da bestimmte Strukturen dahinter sind, dass da bestimmte Akteure dahinter stecken, die Entscheidungen und Entwicklungen beeinflusst haben. sprich der Aufklärungsgedanke ist mir im Grunde auch sehr wichtig, das is auch ein Bereich, weniger in dem neuen Projekt, da bin ich noch nicht so drin, sondern hier in meinem ahm etablierten Gebiet, was ich häufig beforsche, ahm, wo es mir einfach darum geht, ahm, dass manche Dinge ahm nach dem neuesten Stand der Wissenschaft gesehen werden. gutes Beispiel: ahm schadet es den Kindern, wenn sie länger gemeinsam unterrichtet werden? keine Ahnung, es gibt 70 Studien, wovon 69 Studien sagen, das ist nicht der Fall. und eine Studie, die wird dann immer hochgezogen, aber mir ist es dann irgendwie wichtig ahm zum Beispiel rüberzubringen, ich sag das jetzt fiktiv, aber es gibt so und so viele Studien, ahm die allesamt das zeigen, dass es kein Problem is, es is in andern Ländern kein Problem is, dass es gang und gäbe is. ähnlich is, das is jetzt nich mein Forschungsfeld, aber inwieweit schadet es - weil es jetzt grad aktuell is-inwieweit schadet es einem Kind, wenns in der Krippe is. und ahm, da find ich das auch wichtig, dass man ein Stück weit Aufklärungsarbeit macht und sagt, es gibt wenige Hinweise, dass das schadet, sonst hätten keine Ahnung, 97\% aller Schweden nen Vollknall, ist aber nicht so. //I: hm// insofern ahm ist die Aufklärung für mich eine der - auch eine der vornehmsten Aufgaben der Soziologie an sich.

\section{Formulierende Feininterpretation}

Thema: Erfolgreicher Wissenstransfer (Z. 311-323)

T5 definiert sehr erfolgreichen wie auch erfolgreichen Wissenstransfer. Sehr erfolgreicher Wissenstransfer äußert sich in der konkreten Umsetzung von Forschungsergebnissen in der Praxis, erfolgreicher Wissenstransfer in der „Aufklärung" der Gesellschaft.

\section{Thema: Soziologie als Aufklärung (Z. 323-341)}

T5 beschreibt die Relevanz des Aufklärungsgedankens für die eigene Arbeit. Diese definiert er überwiegend über empirische quantitative Forschung als eine der ,,vornehmsten Aufgaben der Soziologie an sich“. 


\section{Reflektierende Interpretation}

Thema: Erfolgreicher Wissenstransfer (Z. 311-323):

Argumentation mit dem Hintergrundmodus einer Beschreibung

Für T5 ist Wissenstransfer gleichbedeutend mit der Umsetzung seiner Forschungsergebnisse in der Praxis. Erfolgreich jedoch ist für ihn „,schon allein Aufklärung“. Er konstruiert bei seiner Definition ein Rationalitätsgefälle zwischen der Soziologie und der Praxis (Beck et al., 1989a, siehe auch Kapitel 2.1). Hier also wird die Selbstbeschreibung gleichzeitig auch zu einer Fremdbeschreibung der Gesellschaft: Es geht darum, die „Leute“ aufzuklären, dass bestimmte Dinge nicht „gottgegeben“ sind. Semantisch steht dahinter ein klassischer Aufklärungsbegriff (Religion vs. rationales Wissen). Der Interviewte verweist damit auf den Sonderstatus soziologischen Wissens, das die „Leute“ missioniert.

\section{Thema: Soziologie als Aufklärung (Z. 323-341):}

\section{Argumentation mit dem Hintergrundmodus einer Beschreibung}

In diesem Abschnitt betreibt T5 Grenzarbeit zu anderen Formen des Wissens über Gesellschaft: In den Themengebieten, die er beforscht, ist es wichtig, auf dem ,neuesten Stand der Wissenschaft“ zu sein. Seine Argumentation bezieht sich überwiegend auf empirische Arbeiten. Aufklärung bedeutet für ihn, wissenschaftliche Studien in ihren Kontext einzuordnen und das Wissen weiterzugeben. Daraufhin kommt er zu einer Art Resümee: dass diese Art der Aufklärung ,eine der vornehmsten Aufgaben der Soziologie“ ist. Die Rhetorik, die er wählt, ist sehr eindringlich, womit er seiner Aussage besonderes Gewicht gibt. Gleichzeitig hebt er jedoch hervor, dass dies nicht die einzige Aufgabe der Soziologie ist. Wie T3 beansprucht er für seine Aussage keine allgemeine Gültigkeit, sondern spricht nur von sich persönlich.

Interview T1, Zeile 37-82

37 I Was ist denn Ihrer Meinung nach die Aufgabe der Soziologie?

38 T1 Ähm (.) hab ich glaub schon so'n bisschen angedeutet, mittlerweile würde

39 ich das auch als-deutlicher sehen als damals, damals war das mehr so intu-

40 itiv. ahm, also ich finde schon äh, dass ne Aufgabe äh der Soziologie ist,(1)

41 Gesellschaft zu beobachten und die Beobachtungen ja - [Name der Person]

42 hat gerade einen schönen Artikel dazu in der Hauszeitschrift der Soziologie

43 geschrieben, also - obwohl ich da'n bisschen von ihm abweiche, also der

44 sagt ja, man solle die Beobachtungen der Leute beobachten und schauen also was denken eigentlich Menschen über Gesellschaft, über soziale Beziehungen, soziale Verhältnisse, soziale Zusammenhänge, ähm wie reflektieren 
sie sie, wie verhalten sie sich praktisch gegenüber Dritten und äh sozialen Institutionen usw. ähm und meines Erachtens ist aber jetzt nicht nur die Beobachtungen der Handelnden ähm ne Aufgabe der Soziologie, sondern ähm ich würde da ergänzen, dass die Soziologie eigentlich auch ähm (1) kritisch infrage stellt das Gewordene ähm, ob das ähm sozusagen alternativlos is, ja, oder ob das notwendig so gekommen is, oder ob's eher zufällig so gekommen is, oder wie es kam, ja, dass das, was wir heute als gesellschaftliche Verhältnisse erleben auch so geworden is. und dieses Gewordensein, ja? dieses Historische von gesellschaftlichen Verhältnisse und gleichzeitig darüber auch zu wissen, es gibt Spielräume ja, der Entwicklung gesellschaftlicher Verhältnisse, ahm, das deutlich zu machen, das herauszuarbeiten, und insofern auch immer Potenziale möglicher Andersartigkeit von Gesellschaft ahm herauszustellen, finde ich ne ganz wichtige zusätzliche Aufgabe der Soziologie. und da würd ich glaub ich über [Name der Person] in dem Sinne hinausgehen, wenn er sagen würde auch eine kritische Soziologie is eigentlich die, die die Kritik die's in Gesellschaft an den gesellschaftichen Verhältnissen gibt, ja? von den ganz normalen Leuten, die die beobachtet und dokumentiert

$$
\text { I } \mathrm{L}_{a h a}
$$

T1 ja, und dann womöglich noch sagt, wie müssten institutionelle Verhältnisse geordnet sein, damit Kritik an der Gesellschaft durch, ja äh, (.) Nichtsoziologinnen, die er dann ,,die Leute“ nennt, überhaupt möglich wird. und ich würde denken, dass es- dass die Soziologie zwar keine normative Position einnehmen sollte als Wissenschaft, dass sie sagt, ,, so oder so müssen gesellschaftliche Verhältnisse gestaltet sein “, aber über die mögliche Andersartigkeit, und über so ne-das is glaub ich so nen Klassiker der kritischen Theorie zu sagen, es gibt unrealisierte Potenziale in dieser Gesellschaft, ja? ahm und die aufzuzeigen, und wie die dann von den Leuten selbst gestaltet werden, das glaube ich auch sollte die Wissenschaft tunlichst den Leuten überlassen, ja? aber zu sagen, es gibt Unrealisiertes, also es gibt Mögliches, was in dieser Gesellschaftsverfasstheit möglich wäre, ja? und es muss nich(2) zehn Prozent Arme geben ja, oder es muss nich so ungleiche Turns of Trade ahm zwischen globalem Norden und Süden geben, ja? und es muss nich äh ständig weitergehen mit dem Klimawandel, ja? das finde ich wichtige Potenzialitäten, die aufgezeigt werden können durch soziologische Beobachtung. 


\section{Formulierende Feininterpretation}

Thema: Die zusätzliche Aufgabe der Soziologie (Z. 38-60)

In diesem Abschnitt legt T1 dar, wie er die Aufgabe der Soziologie einschätzt. Er kontrastiert diese Position mit der Meinung eines Fachkollegen und geht gleichzeitig darüber hinaus. Er weist der Soziologie die Aufgabe zu, „Potenziale möglicher Andersartigkeit“" der Gesellschaft herauszuheben.

\section{Thema: Soziologie und ihre Normativität (Z. 60-82)}

Im nächsten Abschnitt differenziert er seine Aussage weiter. Es geht ihm darum, die Soziologie gegen eine normative Position abzugrenzen. Er argumentiert mit der kritischen Theorie, die er für seine Position einer Soziologie der Potenzialitäten bemüht.

\section{Reflektierende Interpretation}

Thema: Die zusätzliche Aufgabe der Soziologie (Z. 38-60): Argumentation mit dem Hintergrundmodus einer Bewertung

Zunächst fällt an diesem Abschnitt auf, dass er von unzähligen Satzbrüchen geprägt ist. T1 bricht immer wieder ab und setzt neu an. Diese Satzstruktur beinhaltet viele unterschiedliche Argumentationsstrukturen und Legitimierungsansätze für die eigene Position. Angefangen mit der Einleitung, die einen Kontrast aus früher (=intuitiv) und heute (=stärker argumentativ) bildet. Hier bezieht er sich zunächst auf die Position eines Fachkollegen, dessen Meinung er teilt: Soziologie als Beobachtung der Gesellschaft und der Beobachtungen „der Leute“. Diese Person wird einerseits als „Verbündeter“ dargestellt, von dem sich T1 dann andererseits abgrenzt. Diese Abgrenzung ist jedoch mit vielen ,„ähms“ und sehr vorsichtig formuliert. Hier wie auch im weiteren Verlauf werden Parallelen zur Frankfurter Schule sichtbar, die das, was geworden ist, ebenfalls infrage stellt. Es ist jedoch ebenso eine große Geschichte über die Gesellschaft und die gesellschaftlichen Verhältnisse, denn die Frage ist, ob die Soziologie beurteilen kann, inwiefern das „Gewordene“ zufällig, alternativlos oder notwendig so geworden ist. Hier weist der Interviewte der Soziologie einen großen Erklärungswert für gesellschaftliche Verhältnisse zu, ohne dies wiederum kritisch selbst infrage zu stellen. Diese „Potenziale möglicher Andersartigkeit“ benennt er dann als wichtige zusätzliche Aufgabe der Soziologie. Wie T5 argumentiert er, dass dies nicht die einzige ist. Diese Art der Positionierung ähnelt den Aussagen von T5. Denn auch T5 ist auf die Aufdeckung möglicher Andersartigkeit von Gesellschaft eingegangen, die er dann mit „Aufklärung“ definiert. Doch es gibt einen entscheidenden Unterschied zwischen den beiden Darstellungen. T1 legt seine Position wesentlich theoretisch fundierter und auch zögerlicher dar. Diese theo- 
retische Argumentation kann als eine Hilfskonstruktion bezeichnet werden, die die eigene Position innerhalb eines disziplinären Referenzrahmens durch soziologische Theorien legitimieren soll.

Thema: Soziologie und ihre Normativität (Z. 60-82):

Argumentation mit dem Hintergrundmodus einer Beschreibung

In diesem Abschnitt betreibt T1 Grenzarbeit zu seiner eigenen normativen Position. Seine Aussage zeigt die Zerrissenheit des normativen Anspruchs, den T1 durch seine gesellschaftliche Kritik äußern möchte (Armut, Klimawandel), aber auch die Grenze, die die Soziologie zu ihrem Untersuchungsgegenstand einzuhalten hat. Für die Grenze wird in diesem Abschnitt über die normative Position argumentiert, die die Soziologie als „Wissenschaft“ nicht einnehmen darf. Auch hier sind die Satzkonstruktionen zerrissen und es wird eine mögliche Kritik an Normativität bereits antizipiert und in die Argumentation einbezogen. T1 rekurriert auf zwei Hilfskonstruktionen: den disziplinären Referenzrahmen, der eine normative Kritik an Gesellschaft möglich macht (die kritische Theorie), und die sich daraus ableitende Soziologie der Potenzialitäten. Soziologie bleibt damit wie auch bei T5 - mit einem Sonderstatus behaftet, der sich durch ein Rationalitätsgefälle zwischen soziologischem Wissen und anderem Wissen auszeichnet. Man kann von einer Soziologie im Wissensmodus sprechen: Die Soziologie stellt Reflexionswissen zur Verfügung, aber kein Wissen für mögliche Handlungen. Denn was sie tun sollen, das hat die Soziologie dann „tunlichst den Leuten“ zu überlassen. Es wird also ein starker Kontrast aufgemacht zwischen Gestaltung der Gesellschaft und Wissen über eine mögliche Andersartigkeit von Gesellschaft.

Interview T6, Zeile 161-177

161 I [...] da gibt es ja vielleicht auch unterschiedliche Ansichten innerhalb der $162 \quad$ Soziologie?

163 T6 Falsche und richtige. [...] die Falschen sind die, die meinen, sie müssen hel164 fen oder wollen helfen oder können helfen //I: hm// das ist, wenn- wenn- dadann machen Soziologen schlechte Sozialarbeiterei. also ich bin da sehr 166 entschieden schon immer gewesen, ähm und da bin-da seh ich mich wirklich in einer guten Tradition sagen wir mal von der Chicago School //I: hm//

168 Robert E. Park, ja, äh@(.)@ich-ich bin kein verdammter Sozialarbeiter

169 //I: hm// äh ich hab-ich tret-die Soziologie hat nicht die Aufgabe, äh die Welt zu verbessern //I: hm// sie hat die Aufgabe, sich anzugucken wie die

171 Welt funktioniert, was die Leute tun, ne und manchmal machen Leute ganz 

komische Dinge //I: $\mathrm{hm} / /$ und manchmal will man helfen und dann stellt man fest, die Helferei war eigentlich das Gegenteil von dem, was man wollte oder äh die Leute sind nicht so wie man sie sich's vorgestellt hat oder was auch immer. Also ich halt das für eine- für einen unglaublichen Vorteil unseres Faches, eben nicht diese Verpflichtung zu haben, da muss aber am Ende was rauskommen.

\section{Formulierende Feininterpretation}

Thema: Was nicht Aufgabe der Soziologie ist (Z. 163-170)

In diesem Abschnitt legt T6 seine Position dar, welche Aufgabe die Soziologie nicht hat: Sie soll nicht die Welt verbessern.

Thema: Die Aufgabe der Soziologie (Z. 170-177)

Im nächsten Abschnitt hingegen definiert er, welche Aufgabe die Soziologie hat: „sich anzugucken wie die Welt funktioniert“.

\section{Reflektierende Interpretation}

Thema: Was nicht Aufgabe der Soziologie ist (Z. 163-170):

Bewertung mit dem Hintergrundmodus einer Argumentation

Interessant ist in diesem Abschnitt, wie rigoros T6 zwischen den „Richtigen“ und den „Falschen“ trennt. Hier weicht er deutlich von den vorsichtigen und nur individuelle Gültigkeit beanspruchenden Selbstbeschreibungen der anderen Interviewpartner zur Aufgabe der Soziologie ab. Diese Einteilung in richtig und falsch setzt sich weiter fort. Wie bereits in Abschnitt 4.1.1.2.1 argumentiert T6 auch hier mit dem Antiorientierungsmuster einer „helfenden Soziologie“. Soziologie wird also scharf gegen Sozialarbeit abgegrenzt. Auch hier zeigt sich, vielleicht in seiner stärksten Ausführung, das Orientierungsmuster der Soziologie im Wissensmodus. Der Handlungsmodus, in den die Soziologie durch „Helfen“ kommen könnte, wird radikal zurückgewiesen. T6 begründet dies selbstreferenziell, indem er sich in einer Theorietradition verortet. Diese Verortung ist symbolträchtig: Die Chicago School als frühe soziologische Schule, die starke Grenzarbeit zwischen sozialreformatorischen Bewegungen (vertreten beispielsweise durch Jane Addams) und der universitären Soziologie (allen voran vertreten durch Robert E. Park) betrieb. Der Bezug auf eine „wirklich gute Tradition“ unterstreicht hier die Legitimität seines Arguments. Die Purifikation, die die universitäre Chicago School von den sozialreformatorischen Ansätzen betrieben hat, wird hier indirekt auch auf T6 übertragen. Dies wird sogar noch gesteigert durch den Ausruf ,ich bin kein verdammter Sozialarbeiter“. Hier schwingt deutlich Emotionalität mit. Dann geht T6 wieder vom Persönlichen ins Allgemeine: 
Die Soziologie hat nicht die Aufgabe, die Welt zu verbessern. Das Statement, das er hier abgibt, ist also zunächst ausschließend; es beschreibt die Aufgabe, die die Soziologie nicht hat.

Thema: Die Aufgabe der Soziologie (Z. 170-177):

Argumentation mit dem Hintergrundmodus einer Bewertung

Im Gegensatz dazu geht T6 in diesem Abschnitt auf die Aufgabe der Soziologie ein, die bereits mehrfach in den Interviewpassagen angeklungen ist: die Beobachtung der Gesellschaft. Wieder kommt hier das Motiv des Helfens auf. Es scheint, als hätte T6 bereits negative Erfahrungen in der Hinsicht gemacht, weil er angibt, dass „man helfen möchte“, dies aber zum genauen Gegenteil führen kann, also zu nicht-intendierten Nebeneffekten. Hier wird neben einer Ablehnung der Soziologie im Handlungsmodus eine Angst vor einem falschen Agieren deutlich, die sich durch "falsche“ Hilfe ergeben kann. Letztlich zeigt sich das auch in dem Abschlussstatement, da die Soziologie eben nicht auf eine Handlung verpflichtet ist.

\section{Orientierungsmuster "Die Aufgabe der Soziologie“}

In der Analyse der Interviewsequenzen kam deutlich zum Vorschein, wie unterschiedlich 1. die Interviewpartner die Aufgabe der Soziologie definiert haben, 2. wie sie diese rhetorisch begründet und 3. welche Reichweite sie ihrer Argumentation zugewiesen haben. Die Interpretationsoffenheit der Soziologie spiegelt sich in den unterschiedlichen Selbstbeschreibungen. Zudem zeigt sich auch hier der Doppelcharakter der Soziologie dadurch, dass ihr unterschiedliche Zwecke zugewiesen wurden: von einer Anwendung in der Gesellschaft bis hin zu einer selbstreferenziellen Disziplin.

\section{Soziologie im Dienst der Praxis}

T3 stellt die Soziologie klar in den Dienst der Praxis. Dies lässt sich unter anderem durch seine Biografie erklären, die sich durch eine anwendungs- und projektbezogene Karriere auszeichnet:

„Ich habe nie ein zentrales Theorieinteresse entwickelt. Für mich waren Theorien immer mehr Tools, um Muster zu erkennen, um reale Entwicklungen einzuschätzen, um Defizite aufzudecken, aber ich würde nicht sagen, dass ich je ein großes Interesse an einer theoretischen Weiterentwicklung hatte. Das hat sich nie entwickeln können aus Perspektive dieser Projekte, die ich hatte.“ (T3, Z. 69-74). 
Daraus ließe sich die Argumentation ableiten, dass Soziologie als Grundlagenforschung im Sinne einer theoretischen Weiterentwicklung nicht unbedingt im Fokus liegt und Karriereverläufe und Sozialisationsprozesse Einfluss auf die Definition der Aufgabe der Soziologie nehmen können. Die Aufgabe der Soziologie wird auf der sozial-politischen Ebene begründet, und zwar mit der Legitimation vor dem Steuerzahler. Der Soziologie wird in Hinblick auf ihren epistemologischen Status eine Sonderstellung zugesprochen: Die rhetorische Grenze verläuft zwischen Naturwissenschaften und Soziologie. Den Naturwissenschaften wird die Produktion von „hartem“ Wissen attestiert (und somit auch von sinnvoller Grundlagenforschung) und der Soziologie die Produktion von „weichem“ Wissen, das unverbunden nebeneinander steht.

\section{Soziologie als Aufklärung}

T5 und T1 verbindet, dass sie die Aufgabe der Soziologie in der Aufklärung sehen. Beide führen die klassischen Argumente der Aufklärung ins Feld, dass die Wissenschaft „die Leute“ mit rationalem Wissen missioniert. Sie unterscheiden sich aber in der Art und Weise, wie sie den Aufklärungsgedanken darstellen. Während T1 eine starke Selbstvergewisserungsrhetorik nutzt, um seine Position durch einen disziplinären Referenzrahmen zu legitimieren, argumentiert T5 stärker über eigene Vorlieben und die Autorität empirisch angeleiteter Forschung als „,neuesten Stand der Wissenschaft“. Diese Autorität legitimiert auch die entsprechenden Implikationen, die eine solche Aufklärung mit sich bringt. Dahingegen betont T1 den Begriff der Normativität. Normativität relativiert er, indem er das von der Soziologie produzierte Wissen als „Potenziale möglicher Andersartigkeit" darstellt.

\section{Soziologie als handlungsentlastete und selbstbezügliche Disziplin}

T6 konstruiert die Soziologie als eine handlungsentlastete Disziplin. Hier grenzt er sich von einem Helferbegriff ab (,Sozialarbeiterei“) und schreibt der Soziologie eine beobachtende Rolle zu. Im Gegensatz zu den anderen Darstellungen relativiert er seine Aussagen nicht mit einer individuellen Perspektive, sondern bedient sich einer Rhetorik der Allgemeinheit („die Soziologie“). Die Aufgabe der Soziologie wird in diesem Kontext in einer beobachtenden Perspektive verortet. Auch T1 bedient sich einer solchen Rhetorik, wenn er von der soziologischen Community im Allgemeinen spricht: 
schon also also an dieser Frage wie öffentlich soll die Soziologie sein und wie stark soll sie sich positionieren //hm// als eine die- und sei es nur- zur Kenntnis gibt, welche Möglichkeiten einer alternativen Gestaltung bestünden, in Bezug darauf unterscheiden sich die Positionen in der Soziologie sehr stark. //hm// Ahm und ich glaub es gibt schon nicht wenige Kollegen und Kolleginnen, die sagen, äh Soziologie lebt eigentlich davon, dass sie in ner gewissen Weise auch selbstbezüglich is, $/ / \mathrm{hm} / / \mathrm{ja}$, also als Wissenschaft hat sie ihre eigenen Wissenschaftskriterien äh und sie lebt zum Beispiel auch nicht davon, dass sie jetzt jedem auf der Straße verständlich is, ja? //hm// Ähm, sondern, sie hat sozusagen ne ne äh (1) Funktion in in der Selbstbeschreibung von Gesellschaft, und da muss sie möglichst reflektiert sein ja und möglichst ahm dann auch wissenschaftliche Fortschritte- $\circ($ ) $\circ$ und ahm ob man das jetz in ne breitere Öffentlichkeit spielen kann oder nich, is dann kein zentrales Kriterium für die Güte von Soziologie als Sozialwissenschaft.“ (T1, Z. 84-99)

\section{Individuum vs. Disziplin}

Auffallend ist, dass die Interviewpartner in den meisten Fällen keine allgemeingültige Aussage zur Aufgabe der Soziologie treffen. Die Aussagen werden rhetorisch als ausschließlich eigene Meinung dargestellt und damit auch individualisiert. Das oben stehende Zitat kontextuiert diesen Umstand recht anschaulich. T1 äußert sich ähnlich wie T6, dass die Aufgabe der Soziologie in der „Dauerbeobachtung der Gesellschaft“ besteht. Diese Positionierung im „disziplinären Konsens“ mag ein Grund dafür sein, dass sich T6 in seiner Äußerung zur Aufgabe der Soziologie allgemein und nicht individualisiert positioniert. Im Gegensatz dazu sind insbesondere T3 und T1 sehr zurückhaltend in ihren Aussagen: T3 betont mehrmals explizit, dass dies seine Meinung ist. T1 wiederum kontrastiert seine Feststellungen mit denen eines Fachkollegen und grenzt sich von diesen ab. Auch T5 macht klar, dass sein Statement nur für ihn gilt, indem er betont, dass Aufklärung für ihn eine der wichtigsten Aufgaben der Soziologie ist. Die Selbstbeschreibungen werden also rhetorisch so formuliert, dass sie nicht einen Konsens oder eine dominante Selbstbeschreibung der Soziologie widerspiegeln. Im Gegenteil: Es zeigte sich eine gewisse Zurückhaltung, die fast schon ängstlich besetzt ist, Aussagen für die gesamte Disziplin zu treffen. Dies spiegelt sich auch in obigem Zitat wider: Soziologie als Dauerbeobachtung der Gesellschaft, methodisch abgesichert und intersubjektiv nachvollziehbar - das entspricht Kriterien der Soziologie als Wissenschaft. Diese Zuordnung zum Wissenschaftssystem ist konsensfähig. Die Rolle der Soziologie in der Öffentlichkeit - sei es im Sinne einer Soziologie im Dienst der Öffentlichkeit oder einer Soziologie als Aufklärung, befindet sich jedoch nicht im disziplinären Kern. Es ist also zu vermuten, dass gerade diese Positionierung auch eine Positionierung am Rand der Soziologie bedeutet und dass sich T3, T5 und T1 deshalb in der Reichweite und 
Vorsicht ihrer Aussagen zurückhalten. Sie beschreiben hier folglich nicht die Aufgabe der Soziologie, sondern eine Aufgabe der Soziologie, die in ihrer Darstellung auf individuellen Meinungen beruht und mehr oder weniger autoritätsbzw. legitimierungsbedürftig ist. Was sich hier also beobachten lässt, ist eine Ausdifferenzierung der Selbstbeschreibungen auf unterschiedlichen Ebenen: einer individuellen und einer disziplinären Ebene. Diese Ausdifferenzierung veranschaulicht die Uneinigkeit oder auch Unsicherheit, mit der Soziolog(inn)en es zu tun haben, wenn sie Aussagen über die Disziplin treffen.

\section{Soziologie im Wissensmodus}

Zudem wird in den Selbstbeschreibungen eine Grenzziehung zwischen einer Soziologie im Handlungsmodus und einer Soziologie im Wissensmodus deutlich. Diese Selbstbeschreibung beinhaltet gleichzeitig eine Fremdbeschreibung dessen, wie die Interviewpartner ihren Untersuchungsgegenstand definieren, und dementsprechend auch dessen, wie weit die Soziologie in ihren Analysen gehen darf. Präzisiert wird dies insbesondere in den Aussagen von T1, T5 und T6. T6 distanziert sich deutlich von einer Soziologie, die handelnd in das soziale Geschehen eingreift und die er abwertend als „schlechte Sozialarbeiterei“ bezeichnet. In einem zweiten Schritt macht er klar, dass gerade die Soziologie nicht die Verpflichtung habe, handelnd tätig zu werden. Auch T1 und T5 machen deutlich, dass das Wissen der Soziologie durchaus einen gewissen Einfluss auf „die Leute" haben kann, jedoch letztlich nicht als Handlung selbst wirksam werden darf („,das soll die Soziologie tunlichst den Leuten überlassen“). Die Soziologie kann also mit T1, T5 und T6 als eine Wissenschaft beschrieben werden, die im Wissensmodus, jedoch nicht im Handlungsmodus ihre Legitimität findet.

\subsubsection{Soziologie und Werte - ein schwieriges Verhältnis oder: Von der Wertneutralität der Soziologie}

Bereits im historischen Problemaufriss (Kapitel 2.1) wurde erläutert, wie zentral die Frage nach dem Werturteilsstreit frühe Debatten in der Soziologie prägte. Deshalb wurde in den Interviews direkt nach der Wertneutralität der Soziologie gefragt. Dies lenkt das Interview klar in eine Richtung und zielt auf einen innerdisziplinären Diskurs ab, mehr noch, auf eine disziplinäre Selbstbeschreibung umso klarer konnte dadurch zwischen den Interviews kontrastiert werden. Im Folgenden werden drei Interviewabschnitte miteinander verglichen, die unterschiedliche Positionierungen in Hinblick auf die „Wertneutralität der Soziologie" enthalten. 


\title{
Analyse ausgewählter Interviewabschnitte
}

\author{
Interview T8, Zeile 470-516
}

470 I Ähm ich würd' auch ganz gerne noch mal auf das äh Stichwort ähm wertneutrale Soziologie eingehen, [...] ähm wie- wie ist- wäre neutrale Soziologie zu verstehen, gibt es sie?

480 T8 geht doch nicht. also man- man braucht irgendeine Art sozialmoralischer

T8 Na ich finde die Weber'sche Lösung eigentlich immer noch richtig, nämlich die strikte Unterscheidung zwischen dem Wertverhältnis und dem Werturteil. //I: hm// also natürlich kann Soziologie ohne ein Wertverhältnis nicht existieren. also wenn sie sozusagen, welches Thema sie anpacken, hat damit zu tun, welche Wertpräferenzen sie als Soziologin oder Soziologe haben, also ich mein' ohne dem ist ja irgendwie Quatsch

$\mathrm{L}_{j a}$

1

$$
\text { Sensibilität und diese sozialmoralische Sensibilität ist unterschiedlich bei }
$$
Soziologinnen und Soziologen, hat auch was mit Generationen zu tun. also die Frage, also sozusagen die sozialmoralische Sensibilität von äh Dahrendorf, von Scheuch, von Lepsius ist eine völlig andere als von-von mir und Leuten in meinem Alter //I: hm// und das hat etwas mit Werthaltungen zu tun, weil bei uns eher so ne postideologische Dimension ne große Rolle spielt, aber natürlich in der Art und Weise zu forschen, muss man strikt werturteilsfrei sein, //I: hm// sonst kommt man genau, grade jetzt die Bildungssoziologie zeigt mir das wunderbar, die ist meiner Ansicht nach zu stark an Werturteilen orientiert, deshalb verliert sie ihre Neutralität. $\mathrm{Hm} \mathrm{hm}$ und wie kann man strikt wertneutral forschen?

T8 Da gibt's methodische Möglichkeiten, die ähm etwas damit zu tun haben, dass man versucht, also wenn Sie's mal von der quantitativen Seite her angehen, wenn Sie große Datenmengen haben, brauchen Sie irgendwelche plausiblen Modelle und Modelle müssen erstmal, quasi können nur sinnvoll entwickelt werden, wenn Sie eine-wenn sie irgendwie ne Werturteilsfreiheit im Herangehen haben, also dass Sie sagen, irgendwie das- will ich dieses Element für-Modell will ich jetzt nicht nehmen, weil's mir nicht gefällt, das führt zu nichts. also die- die Modellfrage können Sie eigentlich, passende Modelle können Sie nur in einer gewissen Haltung der Werturteilsfreiheit benutzen und Sie können auch nicht ähm-also das ist sozusagen die methodische Seite und die begriffliche Seite, das hatte ich Ihnen eben schon angedeutet, die Soziologie ist schon in Gefahr, Ressentiment-Wissenschaft zu sein, indem-Sie verstärken die Ressentiments der Gesellschaft gegen die 
Ungleichheit. //I: hm// wir haben sozusagen so viele Leute, die-der Reichtum koppelt sich ab usw. und da ist die methodische Frage, ja, stimmt das eigentlich alles und ist- haben wir nicht eher mit $10 \%$ von Doppelverdienerhaushalten zu tun, denen es relativ gut geht. Es ist nicht die Frage von hohen Kapitaleinkünften, //I: hm// der Reichtum ist eigentlich strukturell derselbe wie vor 30 Jahren, da hat sich nicht viel dran geändert. also solche Dinge die können Sie nur in Werturteilsfreiheit klarkriegen und da glaub' ich ist eher wieder würd' ich sagen fast wieder eine Werturteilsfreiheitsmentalität wichtig, also un- sozusagen unbekümmerter zu sein //I: ja, hm// die Soziologie braucht auch, das ist das, was Max Weber die Jugendlichkeit der Psy-der Soziologie nennt, das ist so ne Art Unbekümmertheit, das- wenn sie das verliert, ist- ist es nicht gut.

\section{Formulierende Feininterpretation}

Thema: Die Unterscheidung zwischen Wertverhältnis und Werturteil (Z. 473-490)

In diesem Abschnitt legt T8 seine Position zur wertneutralen Soziologie dar. Er rekurriert auf Webers Unterscheidung zwischen Wertverhältnis und Werturteil und hebt die Notwendigkeit eines Wertverhältnisses in Form einer sozialmoralischen Sensibilität ebenso hervor wie die einer strikt werturteilsfreien Forschung.

\section{Thema: Möglichkeiten der wertneutralen Forschung (Z. 492-516)}

Im nächsten Abschnitt beschreibt er, wie eine wertneutrale Forschung aussehen kann. Diese definiert er über methodische Mittel. „Die begriffliche Seite“ der wertneutralen Forschung wird zunächst über eine Gefahr der Soziologie definiert: Soziologie als „Ressentiment-Wissenschaft“. Indes fordert er eine „Werturteilsfreiheitsmentalität", die der Soziologie unbekümmerte Forschung erlaubt.

\section{Reflektierende Interpretation}

\section{Thema: Die Unterscheidung zwischen Wertverhältnis und Werturteil}

(Z. 473-490): Argumentation mit dem Hintergrundmodus einer Bewertung

T8 nimmt sofort die Erzähleinladung auf, die die Frage nach wertneutraler Soziologie enthält, und argumentiert mit einer disziplinären Referenz: Max Weber. Dabei rekurriert er auf eine Unterscheidung, die dieser getroffen hat, die Unterscheidung zwischen Wertverhältnis und Werturteil. Er bringt damit eine Heuristik in die Argumentation, die in der Soziologie hohe Relevanz besitzt und seine eigene Argumentation unterstützt. Er bezieht sich explizit auf die Soziologie, für deren Forschung es notwendig ist, ein solches Werteverhältnis zum Untersuchungsgegenstand aufzubauen. Er präzisiert seine Aussage durch den Begriff ei- 
ner „sozialmoralischen Sensibilität“. Es ist interessant, dass der Begriff der Werte rhetorisch mit dem Begriff der Moral gleichgesetzt wird. Der Duden definiert Moral als sittliche Grundsätze des Verhaltens (Dudenredaktion, 2010). Was jedoch mit „sozialmoralischer Sensibilität" genau gemeint ist, bleibt im Unklaren. Es ist keine Sensibilität, die übergreifend definiert wird. Vielmehr ist sie an bestimmte strukturelle Kriterien gebunden, er nennt Generation als ein Schlüsselkriterium und beschreibt sich selbst, im Gegensatz zu Dahrendorf oder Scheuch, als Teil einer „postideologischen“ Generation. Dennoch weist er der Soziologie neben einer sozialmoralischen Sensibilität die Notwendigkeit einer strikt werturteilsfreien Forschung zu (Negativbeispiel ist die Bildungssoziologie), die durch die Betonung ,strikt“" rhetorisch aufgeladen wird. Er zieht also eine Grenze zwischen dem notwendigen Werteverhältnis von Forschendem zu seinem Untersuchungsgegenstand und einer strikt wertneutralen Forschung.

Thema: Möglichkeiten der wertneutralen Forschung (Z. 492-516):

Argumentation mit dem Hintergrundmodus einer Bewertung

Die Möglichkeiten einer wertneutralen Forschung benennt er auf Nachfrage zunächst methodisch. Hier kann von einer Purifikationsarbeit gesprochen werden, die eigene Werturteile durch ,irgendwelche plausiblen Methoden und Modelle“ vermeiden soll. Dennoch bleibt T8 in der Darstellung, wie das gelingen kann, im Vagen: Das Grundkriterium für die erfolgreiche Entwicklung solcher Methoden und Modelle ist „,irgendwie ne Werturteilsfreiheit“. Hier wird jedoch ein Zirkularitätsproblem aufgemacht, das ungelöst bleibt: Passende Modelle können nur auf Basis von Werturteilsfreiheit entwickelt werden. Diese können jedoch nur sinnvoll genutzt werden, wenn Werturteilsfreiheit vorliegt. Ob jetzt also die Modellbildung Werturteilsfreiheit voraussetzt oder die Nutzung der Modelle Werturteilsfreiheit bedingt, bleibt offen. Neben einer methodischen Seite macht T8 auch eine „begriffliche Seite“ auf: die Soziologie als Ressentiment-Wissenschaft. Dieser Begriff ist eindeutig negativ besetzt und bezieht sich darauf, dass die Soziologie die Ressentiments in der Gesellschaft, was Ungleichheit betrifft, verstärken kann und dass sie durch ihre Reichweite einen negativen Einfluss auf die Gesellschaft ausübt. Deshalb fordert er eine „Werturteilsfreiheitsmentalität“ für die Soziologie. Diese Mentalität, im Sinne einer Gesinnung, Haltung oder Überzeugung, ist jedoch wiederum etwas, das normativ geprägt ist. Auch das damit verbundene ,unbekümmert“-Sein steht in gewissem Widerspruch zu einer Selbstbeschreibung der Neutralität der Forschung, da Neutralität semantisch auch Disziplin und Selbstkontrolle impliziert, Faktoren, die jedoch in seiner Selbstbeschreibung eine geringe Rolle spielen. Wertneutralität wird hier als „Haltung“ expliziert. 


\section{Interview T9, Zeile 266-337}

266 I Also das eine wär' eigentlich auch diese- diese Frage nach-nach diesem

267 ganz klassischen Werturteilsstreit //T9: ja// also und Max Weber, wie kann man- äh wie- wie kann man überhaupt diese Frage oder dieses Postulat, //T9: ja// was er eigentlich aufstellt //T9: ja// befriedigend erfüllen, beantworten?

T9 Also erstens noch mal zur empirischen Sozialforschung, zur sogenannten Normalsoziologie, wie das Burawoy nicht abwertend sagen würde, sondern als Basic von all- Bas- ja, Basic für alles andere. äh die ist insofern immer kritisch äh als sie ja mhm zunächst mal äh z-zweifelt an- an allem, was behauptet wird und sozusagen gesicherte empirische Kenntnis anstelle der Vermutung setzt.

Linsofern ist das ne einfache Außenposition //I: hm, ja// äh wie Boltanski sagen würde, nicht. so, das ist erst mal das- die Basis von allem anderen. ähm wie ist das jetzt mit- mit- mit der Wertung der Parteinahme? also jetzt muss man sicher den alten Weber auch mal anschauen, was der getan hat //I: hm// und schauen Sie sich die politischen Schriften an //I: hm// äh also da ist jetzt überhaupt nichts von (2) ähm Nichtparteinahme zu spüren. also die Art und Weise wie er mit den Sozialdemokraten umgesprungen ist, mit der Sozialdemokratie seinerzeit, das war natürlich unsäglich, ja //I: hm// er selbst hat das, so wird jedenfalls behauptet, so gelöst, dass er in seinen Vorlesungen als Wissenschaftler auf dem Podest stand und wenn er dann, was üblich war, seine politische Philippika losgelassen hat, trat er vom Podest und sprach sozusagen auf dem Boden

das mach' ich auch manchmal, ja

$$
\mathrm{L} \text { zu seinen Studierenden, }
$$
oalso ich mache das dann mit seiner Analogie, ja○. So, ich-ich glaube, dass $f$-folgendes Problem existiert und ich ko- gehe nicht über Boltanski raus im Grunde. äh auf der Basis der einfachen Außenpositionen jetzt der empirischen Sozialforschung äh können Sie Aussagen machen über gesellschaftliche Teilbereiche, aber nicht über Gesellschaft äh in toto. ich behaupte nach wie vor, dass es so was gibt wie Gesellschaft in Gänze, //I: hm// die ist nicht empirisch zu rekonstruieren, das heißt wir sind auf ne Art Ordnungskonstruktion angewiesen, also ne Konstruktion angewiesen von Gesellschaft, wo 
303

304

305

306

307

308

309

310

311

312

313

314

315

316

317

318

319

321

322

323

324 I

325 T9

326

327

328

329

330

331

332

333

334

335

336

337 wir Bestimmtes stilisieren und akzentuieren und anderes nicht. ähm will man Gesellschaft oder Gesellschaftsmodelle, will man etwa ne kapitalistische Gesellschaft als kapitalistische Gesellschaft kritisieren, kommt man nicht umhin, äh sie theoretisch zu konstruieren. da muss man aber die äh Konstruktionsprinzipien offenlegen, //I: hm// ja und äh im Grunde entspringt Wissenschaftichkeit, also das- das würde für mich, noch ein Satz davor, diese Konstruktion gesellschaftlicher Ordnung würde für mich erlauben, ne komplexe Außenposition einzunehmen. komplexe Außenposition heißt, ich ordne die empirischen Forschungen in einen Zusammenhang ein, äh dessen Konstruktionsprinzip, den ich aber kenntlich gemacht habe. //I: hm// ähm so und die Wissenschaftlichkeit entsteht aus einem ständigen Ringen zwischen den empirischen Forschungen und den Konstruktionen, //I: hm// das ist äh ein Prozess, dem sich eigentlich kein Forscher und keine Forscherin entziehen kann, //I: hm// so sie denn Aussagen machen, die über den gesellschaftlichen Teilbereich rausgehen. so und das ist ein-das ist ne-ne-ne schwierige Gratwanderung, die würde aber heißen, dass es die- die vollkommene Werturteilsfreiheit letztendlich nicht gibt. ich bin eigentlich sicher, dass es dem Weber völlig klar war //I: hm// ähm zumal er ja in ner Weise als politischer Soziologe agiert hat, der hat ja einen Verfassungsentwurf geschrieben, ja, beispielsweise, ja, äh also da ist er sozusagen schon im Bereich der beratenden

$\mathrm{L}_{j a}$

LSoziologie, nicht? äh ich- ich glaube, da- dass man auch Weber insofern ernst nehmen muss und das ist ein ständiges Ringen jetzt mit den vielen linken Studierenden beispielsweise, die gezielt nach [Name des Ortes] kommen inzwischen, dass ne-ne Forschung nicht deshalb besser ist, weil sie sich jetzt meinetwegen auf Marx'sche Theorie beruft, //I: hm// ja, dass sie nicht deshalb besser ist, weil sie einen kapitalistischen- äh kapitalismuskritischen Anspruch hat oder so was, ja, das-das glaube ich nicht und auch äh es gibt auch keinen Zusammenhang dergestalt, dass ne öffentliche Soziologie ne-ne marxistische Soziologie oder so was sein muss, //I: hm hm// ja? Das geht bei uns ein bisschen verloren tendenziell, äh das heißt Weber, das Spannungsverhältnis, das muss man-also da wäre ich jetzt sozusagen in der aktuellen Debatte in unserem Institut näher an Weber als an manchen sehr schnell, vorschnell parteinehmenden Ansätzen. 


\section{Formulierende Feininterpretation}

Thema: Einfache Außenpositionen (Z. 271-278)

T9 beschreibt mit Rückgriff auf Michael Burawoy und Luc Boltanski die „Normalsoziologie“ als Soziologie der einfachen Außenpositionen.

\section{Thema: Weber als öffentlicher Soziologe (Z. 278-295)}

In diesem Abschnitt beschreibt T9 Max Weber als öffentlichen Soziologen, der selbst Partei genommen hat. Er erläutert, welches Prinzip dieser angewandt hat, um seine Meinungsäußerung zu kennzeichnen. Dann macht er sich das Prinzip Max Webers zu eigen, indem er es selbst anwendet.

Thema: Grundprinzipien der Wissenschaftlichkeit (Z. 295-317)

T9 beschreibt „Wissenschaftlichkeit“ als dialektisches Prinzip zwischen der empirischen Forschung und gesellschaftlichen Konstruktionsprinzipien. Hier bezieht er sich wiederum auf Boltanski und dessen Konzept der ,komplexen Außenpositionen“.

\section{Thema: Werturteilsfreiheit und öffentliche Soziologie (Z. 317-337)}

T9 beschreibt das Erreichen von Wissenschaftlichkeit als Ringen und als Gratwanderung. Letztlich negiert er die vollkommene Werturteilsfreiheit wiederum mit Bezug auf Weber und seine beratenden Aktivitäten, wie das Schreiben eines Verfassungsentwurfs. Er stellt dar, dass die Prinzipien öffentlicher und kritischer Soziologie nicht zwangsläufig mit einer bestimmten Theorierichtung verbunden sind, was den Erwartungen , linker Studenten“ entgegensteht.

\section{Reflektierende Interpretation}

\section{Thema: Einfache Außenpositionen (Z. 271-278): Argumentation}

Im Gegensatz zu T8 geht T9 zunächst nicht auf Weber ein, sondern bezieht sich als Hintergrundfolie für seine Argumentation auf zwei zeitgenössische Soziologen: Burawoy und Boltanski. Burawoys Unterscheidungen zwischen Normalsoziologie, öffentlicher Soziologie (public sociology), kritischer Soziologie (critical sociology) und policy sociology (Burawoy, 2005) dienen T9 immer wieder als Referenzfolie seiner Selbstbeschreibung. Durch seine Äußerung „wie Burawoy nicht abwertend sagen würde" zeigt er seine Vertrautheit mit Burawoy und seinen Konzepten. Dann rekurriert er auf eine weitere Referenzfolie, Boltanski, und darauf, wie dieser mit der Stellung der Soziologie zu ihrem Untersuchungsgegenstand umgeht, indem er einfache und komplexe Außenpositionen konstruiert. Diese beiden Positionen macht sich T9 zu eigen, indem er sie zur Basis für alles andere erklärt. 
Thema: Weber als öffentlicher Soziologe (Z. 278-295):

Argumentation mit dem Hintergrundmodus einer Erzählung

In diesem Abschnitt kommt T9 auf Max Weber zu sprechen, beschreibt diesen jedoch anders als T8 als Soziologen, der sich durchaus durch „Parteinahme“ auszeichnete. T9 weist also nicht auf die epistemologischen Prinzipien Webers hin, sondern auf die Person Weber und seine politischen Aktivitäten. In diesem Sinn relativiert er den „Mythos Weber“, der letztlich geschaffen wird, wenn wie T8 das in seiner Selbstbeschreibung macht - von einer Unterscheidung zwischen Wertbeziehung und Werturteil die Rede ist. Auch hier macht T9 sich Webers Position zu eigen, und zwar nicht Webers wertneutrale, sondern Webers politische Position. Auch dient Weber als Referenz, wenn es um die Unterscheidung zwischen wissenschaftlichem und politischem Wissen während der Vorlesung geht (bei politischen Reden verlässt Weber das Rednerpodest).

\section{Thema: Grundprinzipien der Wissenschaftlichkeit (Z. 295-317):}

Argumentation mit dem Hintergrundmodus einer Bewertung

Auch in diesem Abschnitt bezieht T9 sich auf eine wissenschaftliche Referenzfolie, nämlich in Sachen Umgang mit Werten in der Forschung auf Boltanski. Diese Referenzfolie beschreibt er jedoch nur implizit, indem er auf Prinzipien der Wissenschaftlichkeit eingeht: Wissenschaftlichkeit entsteht aus einem dialektischen Verhältnis zwischen empirischer Forschung und den Gesellschaftskonstruktionen der Forschenden. Diese Konstruktionen sind für T9 normativ geprägt, denn er spricht davon, „die kapitalistische Gesellschaft als kapitalistische Gesellschaft zu kritisieren“, was sich auch, im Sinn einer Fremdbeschreibung der Gesellschaft, in ihrer theoretischen Konstruktion äußert. Diese den Konstruktionsprinzipien inhärente Normativität kann legitimiert werden, indem sie offengelegt wird.

\section{Thema: Werturteilsfreiheit und öffentliche Soziologie (Z. 317-337):} Argumentation

T9 stellt den oben genannten Vorgang als „schwierige Gratwanderung“ dar. Die „vollkommene Werturteilsfreiheit“, so schließt er, gibt es nicht. Hier kontrastieren seine Aussagen zu denen von T8, denn er dekonstruiert die Werturteilsfreiheit. Es ist also eine Gegenerzählung zu der Werturteilsfreiheit in der Soziologie. Als Referenzfolie dient dabei wie auch bei T8 Max Weber. T9 betont, im Kontrast zu T8, Webers politisches Engagement und konstatiert, dass Weber die Nicht-Existenz der Werturteilsfreiheit vollkommen klar gewesen sei. Das ist eine sehr interessante Wendung, da Weber ja oft - wie im Fall von T8 - für die gegenteilige Argumentation herangezogen wird. T9 suggeriert mit seinen Äuße- 
rungen, Max Webers Gedankengänge zu kennen, und gibt dadurch seinem Argument rhetorisch Gewicht. Er nutzt die Autorität Webers für seine argumentativen Ziele.

\section{Interview T7, Zeile 432-470}

432 I $\quad H m \mathrm{hm}$. Ähm es gibt ja auch diesen, also jetzt auch noch mal einfach mal Stichwort Werturteilsstreit //T7: hm// und wertneutrale Soziologie, was be-

T7 Ja (3) ja wie soll ich das sagen? also ich äh i- ich bin schon der Meinung, man kann als Wissenschaftler auch ne Haltung äh äh durchaus einnehmen, aber man muss sie eben in gewisser Weise auch explizieren, äh man muss auch in der öffentlichen Kommunikation durchaus äh sozusagen Grenzen und Möglichkeiten der wissenschaftlichen Erkenntnisse immer mit vermitteln, vielleicht auch mal sozusagen den Unterschied zwischen einem empirischen Befund //I: hm// und einer subjektiven Bewertung deutlich machen. ist klar, Wissenschaftskommunikation funktioniert nicht immer so wie wir uns das vorstellen, //I: hm// da gibt's auch durchaus ein Eigenleben, es gibt natürlich auch sozusagen die journalistische Verarbeitungsmaschinerie, darf man auch nicht zu naiv sein, dass man äh zu allem immer gleich irgendwie äh Korrekturen äh anmelden äh kann, aber wenn Wissenschaft äh so was äh so was auch immer mitdenkt, ich glaube, dann würde sich die Form der Wissenschafts-äh-kommen- -kommunikation auch äh auch zwar verändern und dasselbe seh 'ich natürlich auch in der Forschungsarbeit. also die Wahl des Gegenstandes ist natürlich auch schon irgendwie subjektiv eingefärbt, manchmal sogar normativ äh, aber das ist auch legitim zur Motivation einer Forschungsfrage, //I: hm// äh auch in den Naturwissenschaften äh gibt's das oder in der Medizin, dass Leute Dinge erforschen, weil sie jemand mit ner ähnlichen Erkrankung im Umfeld haben oder die Krankheit vielleicht selbst haben, äh warum sollte das ne illegitime äh Motivation äh sein, aber es geht immer so ein bisschen um das Offenlegen und auch äh sozusagen die Vermittlung der Motivation für bestimmte Forschungsthemen und Forschungsfragen, //I: hm// von denen ich denke, dass das irgendwie mit äh mit dazu gehört und äh der Werturteilsstreit ist für mich so ein bisschen ja sozusagen äh bisschen überstrapaziert in kochen@und daraus sozusagen keine äh Frage der Autologiediskussion oder Frage der Erkenntnistheorie äh machen, sondern wirklich äh ne Frage 
465

466

467

468

469

470

des tatsächlich alltäglichen praktischen Umgangs mit der Art und Weise wie wir Wissen generieren oder wie wir Wissen nutzen und dann auch wie wir Wissen weiter-weiter vermitteln und da sind sozusagen die Kontextbedingungen der wissenschaftichen Erkenntnis äh immer etwas, was man irgendwie auch mitführen und mitdenken und manchmal eben auch explizieren muss.

\section{Formulierende Feininterpretation}

Thema: Verhältnis der Soziologie zu ihrem Untersuchungsgegenstand (Z. 435-449)

T7 beschreibt, dass Wissenschaftler selbst eine Haltung einnehmen dürfen, solange diese transparent kommuniziert wird. Er weist auf die Notwendigkeit hin, in der Öffentlichkeit den Unterschied zwischen Empirie und Bewertung deutlich zu machen. Zudem expliziert er, inwieweit auch Wissenschaftskommunikation ein „Eigenleben“ besitzt, das ein Forscher nur bedingt beeinflussen kann.

Thema: Wahl des Forschungsgegenstandes (Z. 449-459)

Er stellt fest, dass bereits die Wahl des Forschungsgegenstandes „subjektiv“ oder „normativ“ geprägt ist. Er geht auf die Legitimität dieser Subjektivität/Normativität ein und zieht einen Vergleich zu den Naturwissenschaften.

\section{Thema: Werturteilsstreit (Z. 459-470)}

Er legt dar, dass er die Debatte über den Werturteilsstreit für ,überstrapaziert“ hält. Für ihn ist diese gleichbedeutend mit erkenntnistheoretischen Debatten, wobei er die Frage der Normativität eher als alltägliche praktische Frage der Wissensgenerierung und der Wissensverwendung betrachtet.

\section{Reflektierende Interpretation}

Thema: Verhältnis der Soziologie zu ihrem Untersuchungsgegenstand (Z. 435-449): Bewertung mit dem Hintergrundmodus einer Argumentation

T7 leitet die Antwort auf die Frage nach wertneutraler Soziologie zunächst ein mit einer Gegenfrage („Ja, wie soll ich das sagen?“), einer langen Denkpause und einer defensiven Rhetorik: „Also ich äh i- ich bin schon der Meinung“. Insbesondere das „schon“ antizipiert einen möglichen Einspruch gegen seine Aussage. Danach setzt er sofort ein „aber“, (,,aber man muss sie eben in gewisser Weise auch explizieren“) und begründet damit auch seine Meinung, dass jede „Haltung“; die ein Wissenschaftler einnimmt, expliziert, also transparent gemacht werden muss. Im Gegensatz zu T8 und T9 führt er keine disziplinäre Referenzfolie an, um seine Argumentation zu untermauern. Das Prinzip der Trans- 
parenz ist ausreichend. Dieses bezieht er nicht nur auf die Wissenschaft, sondern auch auf die öffentliche Kommunikation. Hier differenziert er zwischen empirischem Befund und subjektiver Bewertung. Letztlich könnte durchaus eine Parallele zu Webers Unterscheidung zwischen Sachaussage und Werturteil gezogen werden, die T7 jedoch nicht bemüht. Seine Argumentation steht für sich. Im Gegensatz zu den Ausführungen von T8 und T9 sind seine Aussagen nicht selbstreferenziell, sondern fremdreferenziell - er geht auf die diversen Verarbeitungslogiken wissenschaftlicher Erkenntnisse außerhalb der Wissenschaft ein (Wissenschaftskommunikation). Dennoch argumentiert er selbstreferenziell, indem er beschreibt, dass die Wissenschaft in der Pflicht steht, „Grenzen und Möglichkeiten wissenschaftlicher Erkenntnisse“ zu vermitteln. Die Reichweite wissenschaftlichen Wissens in der Öffentlichkeit wie auch in der Wissenschaft zu relativieren ist ein wichtiger Aspekt seiner Selbstbeschreibung.

\section{Thema: Wahl des Forschungsgegenstandes (Z. 449-459):}

\section{Argumentation mit der Hintergrundkonstruktion einer Begründung}

Wie T8 geht T7 darauf ein, dass die Wahl des Forschungsgegenstandes subjektiv oder normativ geprägt sein kann. Anders als bei T8 jedoch sieht man an dieser Stelle einen rhetorischen Bezug und eine Legitimierung der Subjektivität über die Naturwissenschaften. Während T8 das Wertverhältnis über die Weber'sche Unterscheidung darstellt und mit einem „ohne dem ist ja irgendwie Quatsch“ recht flapsig selbst kommentiert, legitimiert T7 den Wertbezug über einen Vergleich mit den Naturwissenschaften. Er bezieht sich in seiner Rhetorik explizit auf die Legitimität eines solchen Bezugs und stellt dann die Frage, wieso es denn illegitim sein sollte, wenn ein Naturwissenschaftler ein persönliches Interesse an der Erforschung einer bestimmten Krankheit hat. Diese rhetorische Frage lässt keinen Spielraum für ein Gegenargument.

\section{Thema: Werturteilsstreit (Z. 459-470): Argumentation}

Im Gegensatz zu T8 und T9 lässt sich T7 nicht auf eine disziplinäre Debatte zum Werturteilsstreit ein. Dies ist konsistent mit seiner vorhergehenden Argumentation: Er sieht die Frage nach der Wertneutralität der Soziologie letztlich nicht als Frage der Erkenntnistheorie, sondern definiert diese als Frage des „alltäglichen praktischen Umgangs mit Wissen“. Obwohl auch für T8 und T9 letztlich methodologische oder theoretische Konstruktionsprinzipien im Vordergrund standen, die ebenso als forschungspraktische Fragen begriffen werden können, erfolgt die Rahmung der Frage nach Wertneutralität hier grundlegend anders. T7 definiert Werte als Kontextbedingungen wissenschaftlicher Erkenntnis, die explizit gemacht werden müssen. 


\section{Orientierungsmuster „Die Wertneutralität der Soziologie“}

In allen drei Fällen ist deutlich geworden, dass die Argumentation, mit denen die Interviewten sich jeweils zum Werteverhältnis der Soziologie zu ihrem Untersuchungsgegenstand geäußert haben, in unterschiedlicher Weise mit Selbstvergewisserungsrhetorik verbunden war. T8 bezog sich auf die Notwendigkeit strikter Wertneutralität und die Gefahr, dass die Soziologie zur RessentimentWissenschaft wird. T9 hingegen negierte die Möglichkeit einer vollkommenen Werturteilsfreiheit und machte wiederum ein Spannungsfeld zwischen eigenen Werten und Wissenschaftlichkeit auf, das er als „schwierige Gratwanderung“ bezeichnet. T7 gerät bei seiner Argumentation zunächst in die Defensive. Er greift dann auf einen Vergleich mit den Naturwissenschaften zurück, um die Legitimität einer Wertbeziehung der Sozialwissenschaften zu ihrem Untersuchungsgegenstand zu veranschaulichen.

\section{Referenzfolien als Identitätsfolien}

Aus den vorhergehenden Ausführungen ist deutlich geworden, dass es sich um Focussierungsmetaphern handelt, in denen die Frage nach der Soziologie und ihrer möglichen oder notwenigen Wertneutralität auf unterschiedliche Weise behandelt wird. Es haben sich unterschiedliche Selbstbeschreibungen herauskristallisiert, die sich in einem Orientierungsrahmen diverser Referenzfolien bewegen. Wir haben bei T8 und T9 gesehen, dass innersoziologische bzw. auch epistemologische Referenzfolien dazu dienten, die eigene Position zu festigen. Dominierend für beide war die Referenz zu Max Weber. Max Weber als Gründervater der Soziologie und als Protagonist im Werturteilsstreit ist eine Figur, die in besonderem Maße Autorität ausstrahlt. Jedoch differierte die Art und Weise, wie Max Weber für die Argumentation genutzt wurde. Max Weber erscheint als Januskopf (oder Janusargument): T8 verwendete Webers Abhandlungen, um die Notwendigkeit einer strikten Wertneutralität in der Forschung zu unterstreichen. T9 hingegen stellt den öffentlichen Soziologen Weber in den Vordergrund, dessen politische Aktivitäten und sein eigenes vermeintliches Wissen darum, dass Weber klar gewesen sein muss, dass es die vollkommene Wertfreiheit nicht geben könne. T9 konstruiert in diesem Sinn den Anti-Helden Weber und stärkt somit sein eigenes Argument. Interessant ist hier, dass sich wiederum die Interpretationsoffenheit der Soziologie zeigt sowie die Verunsicherung, die der Diskurs um die Wertneutralität der Soziologie mit sich bringt: Einerseits kann eine Galionsfigur der deutschen Soziologie rhetorisch für derart unterschiedliche Argumentationszwecke genutzt werden. Dies macht deutlich, wie Selbstbeschreibungen ausgehend von dem zu bearbeitenden Problem differieren und rhetorische Mittel in der Argumentation strategisch eingesetzt werden. Andererseits wird 
klar, zu welchen Autoritätsfiguren in der Soziologie gegriffen wird, um die eigene Position darzustellen. Dies ist ein Hinweis darauf, dass es wenig feste Referenzfolien oder geteilte „Problemlösungen“ innerhalb der Disziplin gibt. Auffallend ist zudem, dass T9 auf zwei kontemporäre Soziologen als Referenzfolie zurückgriff: Michael Burawoy und Luc Boltanski. Beide haben eine starke Position zu der Frage der Grenze der Soziologie zu ihrem Untersuchungsgegenstand gewählt: Burawoy mit seinem Konzept der public sociology und Boltanskis mit seinem neopragmatischen Ansatz einer Soziologie der Kritik.

Im Gegensatz dazu lehnt es T7 geradezu ab, innerdisziplinäre Referenzen für seine Argumentation zu bemühen. Er rahmt das Problem als ein sozialpolitisches und nicht als epistemisch-kognitives. Seine Referenzfolie ist die eines öffentlichen Diskurses über Wissenschaft, der maßgeblich von der Wissenschaftskommunikation geprägt ist. Obwohl er eine ähnliche Unterscheidung vorschlägt wie Max Weber, nämlich die zwischen Sachaussage und Werturteil, rahmt er das Problem als alltagspraktisches und nicht als erkenntnistheoretisches, wie dies bei T8 und T9 der Fall ist. Zudem nutzt er den Vergleich mit den Naturwissenschaften, um eine Wertorientierung der Soziologie zu legitimieren.

\section{Einbezug der Normativität vs. Ausschluss der Normativität: Diverse Lösungsansätze zur Wertbezogenheit der Soziologie} Alle drei Interviewten schlagen derweil Lösungsansätze vor, wie man letztlich mit der Frage der Wertneutralität der Soziologie umzugehen hat. Während T7 und T9 für maximale Transparenz plädieren (Offenlegung der Konstruktionsprinzipien und Explizieren der Kontextbedingungen der Wissensgenerierung), setzt T8 auf methodologische Purifikationsarbeit, die sich an einem Paradigma der strikten Wertneutralität orientiert. Diese strikte Grenzziehung nach Webers Vorbild kontrastiert mit der Annahme der blurred boundaries zwischen Werten und Forschung: Werte sind nun mal vorhanden und es geht deshalb darum, den Forschungsprozess und die dahinterliegenden Konstruktionsprinzipien transparent zu machen. Die eigene Normativität in der Forschung wird also nicht nur unterschiedlich begründet, wie wir dies im vorherigen Abschnitt gehört haben, sie wird auch jeweils in die eigene Selbstbeschreibung von Forschung integriert oder purifiziert.

Abschließend kann gesagt werden, dass alle drei Interviewten unterschiedliche innersoziologische und externe Referenzfolien anwandten, um ihrer eigenen Position Autorität zu verleihen. Im Gieryn'schen Sinn kann hier von einer Strategie der ,protection“ gesprochen werden. T8 jedoch nimmt in einem zweiten Schritt eine Argumentation vor, die Werturteile aus der eigenen Forschung exkludiert und sich in der Nähe einer wertneutralen Soziologie positioniert. T7 und 
T9 wiederum integrierten die Normativität in ihre eigene Argumentation, man kann also eher von der Strategie „Inklusion“ sprechen. T7 begründet die Legitimität von Werten innerhalb der soziologischen Forschung durch die Naturwissenschaften. T9 konstruiert einen „Anti-Weber“, der in politische Aktivitäten involviert war und um die Unmöglichkeit absoluter Wertfreiheit wusste.

An der Analyse zu den Textabschnitten zur Wertneutralität der Soziologie wird die Doppelstruktur der Soziologie besonders deutlich: Alle Interviewabschnitte zeichneten sich durch eine besondere Legitimierungsrhetorik aus. Zudem gab es unterschiedliche Referenzfolien, mit der Normativität in der Soziologie umzugehen. Auch hier kam keine Einheitlichkeit in den Sinnkonstruktionen zum Vorschein, sondern die Kontingenz und Fluidität der Selbstbeschreibungen in Hinblick auf den adäquaten Umgang mit der Doppelstruktur der Soziologie.

\subsection{DAS VERHÄLTNIS ZWISCHEN DER SOZIOLOGIE UND IHREM UNTERSUCHUNGSGEGENSTAND}

In Abschnitt 4.1 haben wir bereits wesentliche Hinweise für eine Selbstbeschreibung der Soziolog(inn)en in Hinblick auf die Soziologie als Disziplin, ihre eigene Praxis wie auch auf mögliche Grenzziehungen zwischen der Soziologie und ihrem Untersuchungsgegenstand bekommen. Die Sichtweise, die dort untersucht wurde, war überwiegend auf Beschreibungen der Soziologie fokussiert. In Abschnitt 4.2 wird verstärkt das Verhältnis zwischen der Soziologie und ihrem Untersuchungsgegenstand in den Blick genommen, es geht also darum, wie Sozio$\log (\mathrm{inn})$ en das Verhältnis zwischen Soziologie und Nicht-Soziologie definieren.

\subsubsection{Der öffentliche Auftrag der Soziologie}

In den Interviews wurde unter anderem gefragt, ob die Interviewten einen „öffentlichen Auftrag“ der Soziologie bestimmen können. Diese Frage zielte auch auf Selbstbeschreibungen der Soziologie in Hinblick auf ihre Schnittstelle mit der Praxis ab. 